JPL PUBLICATION 78-20

\title{
Large Active Retrodirective Arrays for Space Applications
}

(NASA-CR-156163) LARGE ACTIVE

$N 78-21333$

RETRODIRECTIVE ARRAYS FOR SPACE APPLICATIONS

(Jet Propulsion Lab.) 59 p HC $A 04 / M F$ A01

$\operatorname{CSCL} 20 \mathrm{~N}$

G3/32 $\begin{aligned} & \text { Dnclas } \\ & 14104\end{aligned}$

National Aeronautics and Space Administration

Jet Propulsion Laboratory

California Institute of Technology

Pasadena, California 91103

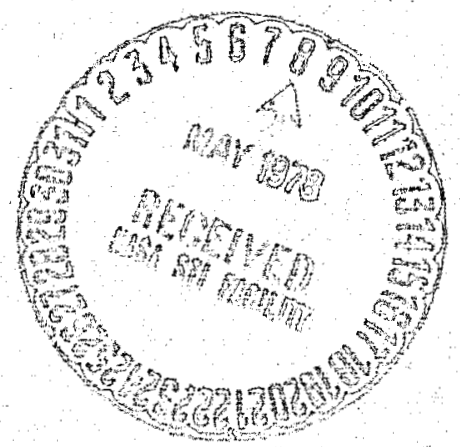


JPL PUBLICATION 78-20

\section{Large Active Retrodirective Arrays for Space Applications}

R. C. Chernoff

January 15,1978

National Aeronautics and Space Administration

Jet Propulsion Laboratory

California Institute of Technciogy

Pasadena, California 9110 ? 


\section{PREFACE}

The work described in this report was performed by the Telecommunications Science and Engineering Division of the Jet Propulsion Laboratory. 


\section{CONTENTS}

I.

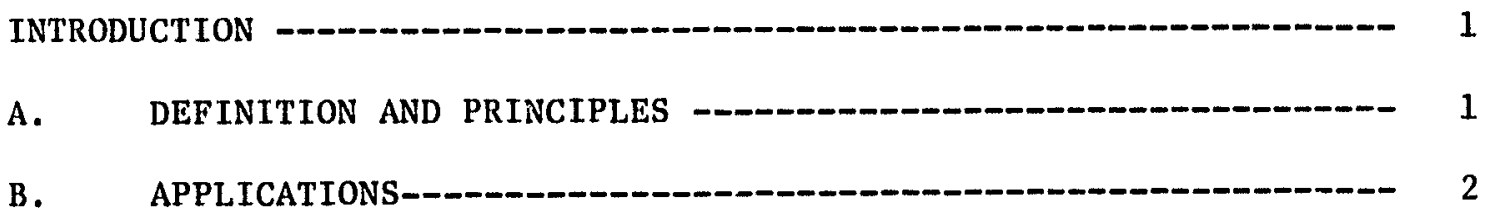

II. ARA DESIGN PROBLEMS AND SOLUTIONS-

A. PHASE REFERENCE DISTRIBUTION

B. PHASE CONJUGATION CIRCUITS- 8

C. POINTING ERRORS IN ARAS-C 13

III. EXPERIMENTAL RESULTS- 21

IV. CONCLUSION- 24

REFERENCES -

APPENDIXES

A. DOPPLER EFFECTS IN ACTIVE RETRODIRECTIVE ARRAYS-- A-1

B. ABERRATION ERROR- B-1

C. TRANSMISSION LINE MISMATCH ERRORS-

\section{TABLE}

1. Phase table for Figure 9

\section{FIGURES}

1. Active retrodirective array 29

2. Central phasing- 30

3. Tree structure for centra11y phased ARA- 31

4. Simple phase conjugating circuit- 31

5. Exact PCC: servoed $\triangle \phi$ type 32

6. Exact PCC: phase-1ocked-1oop type-- 32 


\section{CONTENTS (contd)}

7. Phase reference regenerators (PRRs) for: PCC of Figure 6

8. ARA recelvers- 34

9. Two-tone PCC and PRR 35

10. Doppler pointing error- 36

11. Aberration pointing error- 37

12. ARA breadboard- 38

13. Antenna range set-up - 39

14. Retrodirective array pittern- 40

15. ARA breadboard: effect of line length changes--_-_-_-_--_--- 41 


\begin{abstract}
An active retrodirective array (ARA) electronfcally points a microwave beam back at the apparent source of an incident pilot signal. Retrodirectivity is the result of phase conjugation of the pilot signal received by each element of the array. The problem of supplying the correct phase reference to the phase conjugation circuit (PCC) associated with each element of the array is solved by "central phasing." By eliminating the need for structural rigidity, central phasing confers a decislve advantage on ARA's as large space-borne antennas. A new form of central phasing suitabie for very large arrays is described. ARA's may easily be modifled to sorve both as transmitting and recelving arrays simultaneously. ARA's are particularly suftable as solar power satellite antennas because they are inherently failsafe. Communication satellites and deep space probes are other suggested applications. Two new kinds of exact, frequency translating PCC's are described. Such PCC's provide the ARA with input-output isolation and freedom from squint. The pointing errors sitied by the radial and transverse components of the ARA's velocity, by the propagation medium, and by multipath are discussed. As part of a NASA funded program, a two element ARA breadboard has been built and tested at JPL. Its performance is limited primarily by multipath induced errors.
\end{abstract}


LARGE ACTIVE RETRODIRECTIVE

ARRAYS FOR SPACE APPLICATIONS

\section{INTRODUCTION}

A. Definition and Principles

An active retrodirective array (ARA) transmits a beam towards the apparent source of an illuminating signal called the pilot. "Active" implies that the array produces, not merely refluts, RF power. Retrodirectivity is achieved by retransmitting from each elemptr of the array a signal whose phase is the "conjugat 'if that received by the element. Assuming that the phase of the pilot signal of angular frequency $w$ recelved by the kth element of the array at time $t$ is

$$
\phi_{k}(t)=\omega\left(t-r_{k} / c\right)
$$

where $r_{k}$ is the distance from the $k$ th element to the source of the pilot signal, we define the conjugate of $\phi_{k}$ to be

$$
\phi_{k}(t) *=\omega^{\prime}\left(t+r_{k} / c\right)+\phi_{0}
$$

where $\omega^{\prime}$ is the angular frequenry of the conjugate signal, and $\phi_{0}$ is an arb1trary phase offset which must, however, be constant over the entire array. In order to do this, each element of the array must be equipped with a phase conjugation circuit (PCC) as shown in Figure 1. The phase of the signal received from the kth element by a receiver located at the pilot source $(r=0)$ is, at time $t$,

$$
\phi_{k}(t, 0)=\omega^{\prime}\left(t+r_{k} / c-r_{k} / c\right)+\phi_{0}=\omega^{\prime} t+\phi_{0}
$$

Thus the contributions to the field at $r=0$ from the various elements of the array are all in phase at that point, which means that the beam (or a major lobe if the array is too thin a form a single beam) points toward the pilot source. 
The usual definition of phase conjugation includes only the case $\omega^{\prime}=w^{\prime}$. Our definition is generalized in order to emphasize that $\omega^{\prime}=\omega$ is neither necessary nor desirable; retrodirectivity holds in either case provided only that the propagation medium is non-dispersive, and $\omega^{\prime}=\omega$ is usually to be avoided because of input-output isolation problems.

An ARA can also function as a receiving (i.e., trarking) array. It is easy to show how a single PCC at each element can be used for both functions, receiving as well as transmitting, simultaneously, with little additional equipment. Specific examples will be given in our discussion of PCC's below.

\section{B. Applications}

A1though not a new Idea (the concept was first proposed by W. E. Morrow in 1958 [1], ARA's have yet to be applied practically. A number of experimental systems were built in the early sixties [2], [3], but the ARA activity visible in the Iiterature has been sparse in recent years. ARA's would seem, however, to fit quite naturally into space communication systems requiring highly directive spacecraft antennas. In the following we outline just three of the many possible ARA space applications.

\section{Solar Power Satelite}

Much of the current interest in ARA's is centered about its posslble application to the Solar Power Satellite (SPS) concept of Glaser [4] and Brown [5]. The SPS would be placed in a geosynchronous orbit. Several gigawatts of microwave power, generated by and converted from the de output of huge solar cell panels, would be transmitted to a rectifying antenna ("rectenna") on the Earth's surface. The SPS-rectenna range would be $36,000 \mathrm{~km}$, the rectenna diameter $7.4 \mathrm{~km}$, and the frequency $s$-band $(\lambda \approx 12.5 \mathrm{~cm})$. These parameters plus stringent sidelobe 
requirements imply a transmitting antenna on the SPS about $1.0 \mathrm{~km}$ in diameter. The pointing loss becomes unacceptable if the miss radius exceeds $200 \mathrm{~m}$, which, in angular terms, corresponds to $5.6 \times 10^{-6}$ radians $=1.1$ arc seconds. Because of the cbvious difficulty in mechanically pointing a $1.0 \mathrm{~km}$ drameter antenna to this accuracy, the proponents of the SPS suggest using an ARA for the spacecraft antenna 'Nith the pilot source located at the center of the rectenna.

An equally important reason for using an ARA for the SPS is safety, specifically, the need to protect the public from exposure to the high power beam. Although no beam pointing system is infallible, the ARA would seem to be the most Inherenty reliable system for this application since its retrodirectivity is inseparable from the beam forming process itself. Such pointing errors that are known to exist (discussed in Sect. II) produce only slight (compared to rectenna diameter) mispointing. Moreover, the response time of an ARA is determined by its own dimensions, not by the ground to spacecraft round trip delay as It would be for a conventional closed loop control system. It would, therefore, be of the order of microseconds, not $2 \times 36 \times 10^{6} /\left(3 \times 10^{8}\right)=0.24$ seconds, the round trip delay for an SPS in geosynchronous orbit.

\section{Communication Satellites}

The applicability of ARA's to communication satellites was noted by Hansen [6] and others. Large antennas on communication satellites will be required not only to serve ground receivers with small apertures (as in direct TV transmissions), but also to provide the directivity needed for spectrum conservation.

A communication satellite ARA could use either frequency or time division multiplexing. In a frequency multiplexed version designed to communicate with each of $n$ ground stations, each element of the array is equipped with $n$ phase 
conjugation circuits, and each circuit responds only to the carrier frequency of the pilot signal transmitted by one of the $n$ stations. Information modulated on the carrier of the pliot signal from one of the stations, call it Station $A$, is demodulated and remodulated onto the carriers of Jownlink signals retrodirected to one or more of the other ground stations. Simllarly, information from any of these other stations can be simultaneous1y modulated onto the downlink to A. The average power avallable for each downlink must, of course, decrease with $n$, but the full gain of the array is avallable to each downlink independently of $n$.

If time division instead of frequency misltiplex is used, only one phase conjugation circuit and one recelver is required for each element. However, the bandwidth of that recelver must be $n$ times greater than that of each of the $n$ receivers required in the frequency multiplex case. Depending on the dimensions of the array, the required bandwidth, and the scan angles required to point beams at different ground stations, time delay compensation may be required in order to properly synchronize the data streams transmitted by the various elements [7] Deep Space Probes

ARA's may also be useful as deep space probe antennas. As the (distance) $x$ (data rate) product increases, it will eventually become necessary to use spacecraft apertures too large to be mechanically pointed. Here, however, certain errors proportional to the velocity of the spacecraft relative to the ground station may become important. These errors are discussed in detail in Sect. II below. They are unimportant in geosynchronous satellite, such as the SPS and most communication satellites, because of their small relative velocities, but deep space probes may experience much higher velocities in the course of a 
mission, and this factor may limit the size of the ARA which can be used on such spacecraft.

Deep Space Ground Receivers

We noted above that an ARA can function as a receiving array. Such arrays may be useful for recelving weak signals from very distant deep space probes or as radio astronomy arrays. Since low noise front ends are fairly expensive, such an array would probably consist of a modest number of falrly large antennas rather than a very large number of small elements. Each of the large elements would be mechanically steered to keep the source within its beamwidth. As in communication satellite ARA's, data processing may be ro juired to remove time delay distortion.

\section{ARA DESIGN PROBIEMS AND SOLUTIONS}

A. Phase Reference Distrifution

From (1) and (2) we see that phase conjugation amounts to advancing the phase of an input signal by an amount equal to its delay. The phase conjugation circuit (PCC) must, therefore, be provided with a phase reference against which to measure that delay. If we locate each PCC at its associated ARA element as in Figure 1, then it is clear that we must transmit the phase reference to each PCC from some central source via transwission lines of equal phase delay modulo $2 \pi$. But it may be difficult to do this if the transmission lines are very long. For example, consider the $1.0 \mathrm{~km}$ diameter SPS ARA described above operating at S-band $(\lambda=12.5 \mathrm{~cm})$. If the master phase reference is located at the center of the disk, the transmission lines to elements at the periphery will be $500 \mathrm{~m} 10$ g. If we wish to keep the phase delay in this line constant to within $\pi / 10$ radians, its length must not vary by more than $\pm \lambda / 20 \mathrm{~cm}$, or a relative change no greater than 
$\pm 1.2 \times 10^{-5}$. But this length change would be produced $\mathrm{un}$ an aluminum line by a temperature change of only 0.5 degrees $C$, or by a mechanical stress of only 120 ps1. The results finr other good conductors are simflar. Since we would expect temperature and stress changes far greater than these in this huge structure, it is clear that the required dimensional stability cannot be met with materials commonly used for transmission lines.

While it might be possible to solve this problem with uricommon materials, we can avoid it altogether'by locating all PCC's at the reference source rather than at their respective elements. This method oi providing the phase reference, which Margerum cal1s "central phasing" [8], is 11lustrated in Figure 2, which, for the sake of clarity, shows only two elements of the ARA. The phase reference for this ARA is the pilot signal recelved by the $0-t h$, or reference, element. The pllot signal received by the kth element is transmitted to its associated PCC located at the reference element via the transmission line and diplexer, $2 \mathrm{PLX}$. The PCC conjugates the entire phase delay, 1.e. the sum of the space delay, $\omega r_{k} / c$, and the transmission 1ine delay, $\omega_{k o} / c_{L}$, where $c_{L}$ is the phase velocity of the line, and transmits that conjugate signal back down the same transmission Iine to the kth element, which retransmits it. Its phase at that point is $\omega^{\prime}\left(t+r_{k} / c\right)+\phi_{0}$ which is exactly what it would be were the PCC located at the kth element rather than at tre reference element. Thus the length of the transmission line is immaterial provided only that: 1) the line is dispersionless, and 2) its length is constant with time.

The importance of central phasing lies In the fact that it 1iberates the ARA from the rigid structure which would otherwise be needed in order to realize accurate retrodirectivity. The elements need not be arranged in any particular geometrical pattern, and may, in fact, move about with respect to one another 
provided (as we shall show below) the movement is not too rapid. This remark appl:.es, of course, only to pointing accuracy, not to side lobe levels or other characteristfes of the antenna.

Locating all the PCC's in one sma11 volume very near the reference element may be difficult if the array contains thousands of elements. A modification of the central phasing idea using a tree topology in which the phase reference is regenerated at each node will be required in such large arrays. This is $111 u-$ strated in Figure 3. Each branch of the tree consists of a PCC, located near the node, and an element of the ARA at the end of a transmisiston 1ine. A phase reference supplies all the PCC's connected to a node. At the initial node, this is the reference element of the array. $\Lambda t$ subsequent nodes, it is a phase reference regenerator (PRR). The PRR combines samples of the pilot and conjugate signals at an element to reproduce the original reference. Specific ways of doing this will be described in the discussion of PCC's in Subsection B below.

The dashed boxes in Figure 3 contain all the circuit elements located at a node. Since the signal paths within these node assemblies are unilateral, their phase Jelays must be carefully balanced in order to avoid phase error buildup at successive nodes. In order to assure the stability of the phase delay balance, these assemblies must be uniform and compact. Critical applications may require tem sature stabilization of some active elements.

The number of nodes in a tree is relatively small even for an ARA of several thousand elements. For example, if we have six branches at each node, then a tree of only five nodes suffices for an array of 9331 elements. PRR's are required only at the first through fourth order nodes of this tree. A PRR at a 
fourth order node is the last in a chain of four PRR's connecting the PCC's at that fourth order node to the reference element of the ARA. The error in the value of the phase reference produced by this last PRR is the sum of the errors arising in all the PRR's in the chain. If these errors arise from independent and Identical runaim processes in each PRR, then the probable error of the output of the last PRR is $\sqrt{4(P E)^{2}}=2$ (PE) where PE is the probable error of each PRR. Thus the error buildup due to repeated regeneration of the phase reference is moderate even for large arrays.

\section{B. Phase Confugation Circuits}

The simplest phase coniugation circuit (PCC) is shown in Figure 4. Unfortunately, this circuit is impractical because of isolation problems. Unwanted coupling occurs in three ways: 1) through the imperfect circulator, 2) leakage from the input to the output of the mixer, and 3) by mutual coupling between neighboring elements of the array. Shifting the output frequency may solve the isolation proilem, but we may, depending on how the shifting is done, find that the transmitted beam is no longer retrodirected. For example, the output fraquency of this PCC can be shifted simply by offsetting the frequency of the phase reference by $\Delta \omega$. The result is a sort of approximate phase conjugation which, in a planar ARA, causes a pointing error called "squint" given by

$$
\Delta \theta=-\frac{\Delta \omega}{\omega} \tan \theta
$$

for small $\Delta \omega$, where $\theta$ is the scan angle (angle between direction of arrival of the pilot signal and the normal to the array). $\Delta \omega$ has a practical lower bound due to the imperfect isolation of real diplexer filters. Therefore, $\Delta \theta$ may be too large for applications requiring both very precise pointing and a wide scan range. 
In order to avold both these difficulties we would like to have exact, frequency shifting PCC's, 1.e. PCC's that satisfy our definition, Eq. (2), of phase conjugation where $\omega^{\prime} \neq \omega$. A simple example of such a PCC is shown In Figure 5 (after [9]). The servoed phase shifter must be both bilateral (to allow the pilot and conjugate signals to traverse it in opposite directions) and nondispersive. Since the phase shift in any circuit is affected by reflections at both input and output, the phase shifter must be well matched at both $\omega$ and $\omega^{\prime}$. If it is not, the phase shifter will, in effect, be dispersive. Some mechanical phase shifters (e.g., a coaxlal line stretcher) meet both the bilateral and nondispersive requirements. Electrical phase shifters, such as diode and ferrite phase shifters, meet them to varying degrees, and may be satisfactory when $\omega^{\prime}$ is close to $\omega$.

Note that the conjugate signal need not be coherent with the pilot in this PCC; a free running oscillator, which need not even be particularly stable, suffices as the source of the conjugate signal. However, like the reference ral, it must have the same phase at each PCC.

A new kind of exact PCC is shown in Figure 6. This one uses a phase locked loop which both conjugates the phase and translates the frequency of the input signal. Mixer M2 is an upconverter while M1 is a downconverter. The conjugate relation,

$$
\phi_{1}^{*}=R\left(2 \phi_{0}-\phi_{1}\right)=R \omega\left(t+\left(\frac{1}{c}\right)\left(r_{1}-2 r_{0}\right)\right) \text {, }
$$

where $R=1 /(1-2 / n)$, follows immediately from the phase lock condition. The circuit is practical for $n \geq 4$. Thus the sequence of frequency translating ratios, $R$, starts at 2 and converges to 1 as $n$ increases. Since $R-1$ has to be greater than some small num?, in order to obtain the required diplexer isolation, 
only finitely many ratios of the sequence are usable. For example, if we require $R \geq 1.01$, then we may use any $n$ from $4(R=2)$ to $202(R=1.01)$ inclusive. The loop bandwidth in Figure 6 need be no wider than is required to track out the relative doppler rate between the remote an' reference elements $[10]$.

Figure 6 lends itself very well for use in a receiving array. We need only Insert a clean-up loop between the reference element and the PCC's in the initial node assembly, so that the reference signal applied to these PCC's contains only the carrier phase, $w\left(t-r_{0} / c\right)$, and no modulation. The phase detector in Figure 6 then serves as a demodulator whose output is fed to the data summer (through a delay distortion correcting processor if required) along with the data from the other PCC's.

The phase reference regenerator (PRR) for the circuit of Figure 6 may take various forms. The straightforward approach is illustrated by the circuit shown in Figure $7 \mathrm{a}$ which recovers the phase reference $\phi_{0}$ by the proper combination of the conjugate, $\phi_{1}^{*}=R\left(2 \phi_{0}-\phi_{1}\right)$, and the pilot signal, $\phi_{1}$. Note that three mixers are required here, not just two; we cannot add two signals of the same frequency in a single mixer because the upper sideband would then have the same frequency as the second harmonic of the strong signal. We must add the pilot signal to the output of $M 1$ in two stages, $M 2$ and $M 3$, in order to remove this "degeneracy."

Figure $7 \mathrm{~b}$ shows an indirect method of phase reference regeneration. Here, instead of recovering $\phi_{0}$ from $\phi_{1}^{*}$ and applying it as the phase reference to succeeding PCC's as in Figure 7a, we use the PCC at the first order element to produce a "relative" conjugate,

$$
\phi_{1,2}^{*}=R\left(2 \phi_{1}-\phi_{2}\right) \text {, }
$$


which is then corrected to

$$
\phi_{2}^{*}=R\left(2 \phi_{0}-\phi_{2}\right)
$$

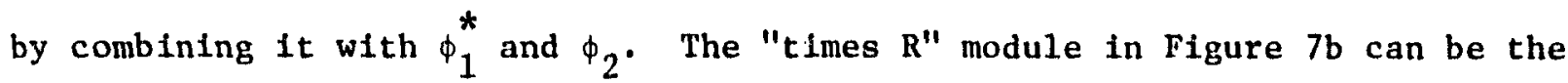
PCC of Figure 6 with $\phi_{2}$ at both inputs.

Before we can go on to the third example of an exact PCC and its associated PRR, we must discuss the problem of recelving the pilot signal. PCC's and PRR's are usually IF circuits, while the pilot is a microwave signal. We must accurately transfer the phase information in the plitot signal to the IF input signal to the PCC. A receiver which does this is called a coherent receiver. The best known coherent receiver is the phase-locked receiver shown in Figure 8a. The average, or slowly varying, part of the pilot signql phase is divided by $n+1$, the ratio of the microwave to IF frequency. Another kind of coherent receiver is shown in Figure 8b. The pilot signal for this receiver must consist of two carrier signals or "tones" transmitted from the same pilot antenna. These tones can be the upper and lower sidebands produced by balanced modulation. The IF output of the "two-tone receiver" in Figure $8 \mathrm{~b}$ is simply a doubled version of the modulation, which contains the phase information in each of the pilot tones divided, again, by the pilot/IF frequency ratio. In Figure $8 b$, we assume that the Lo frequency, $f_{L}$, lies between those of the two pilot tones, $f_{1}$ and $f_{2}$, so that mixer Ml produces the two lower sidebands $f_{1}-f_{L}, f_{L}-f_{2}$. These are separated by band pass filters, and one of them is amplif ed to a level sufficient to serve as the Lo for M2. M2 is an upconverter. Hence, its output phase is

$$
\phi_{1}=\left(\phi_{1}-\phi_{L}\right)+\left(\phi_{L}-\phi_{2}\right)=\phi_{1}-\phi_{2} .
$$


Since

$$
\phi_{1}=\omega_{1}(t-r / c)
$$

and

$$
\phi_{2}=\omega_{2}(t-r / c)
$$

Eq. (4) gives

$$
\phi_{1}=\left(\omega_{1}-\omega_{2}\right)(t-r / c) \text {, }
$$

in fulfillment of our requirement that the IF phase be an accurate representation of the pilot signal phase (or phases) uncontaminated by the phase of extraneous signais (the LO phase in this case).

Note that $f_{L}$ in Figure $8 b$ must not be exactly half way between $f_{1}$ and $f_{2}$, because then $f_{1}-f_{L}$ would be equal to $f_{L}-f_{2}$ which would produce degeneracy in mixer M2. $f_{L}$ need not be between $f_{1}$ and $f_{2}$, but if it isn't, mixer $M 2$ must be a downennverter. The limiter in Figure $8 \mathrm{~b}$ is required to avoid phase offset changes in the mixer $M 2$ produced by changes in its input level.

Provided that a two-tone pilot signal is available, the two-tone recefver can always be used in place of the phase-locked receiver of Figure $8 a$ and will usually be cheaper. Moreover, one can carry the two-tone pilot idea a step further by using TF images of the two pilot tones within the PCC-PRR combination itself, and not just in the coherent recelver ahead of it. Such a circuit is shown in Figure 9. The operation of the circuit can be easily followed with the aid of the accompanying phase table (Table 1). Note that the conjugation is exact; i.e. the output phase is the exact conjugate of either pilot signal. Note also that degeneracy and leakagc in the mixers are avoided because 
$\mathrm{f}_{1}-\mathrm{f}_{\mathrm{L}} \neq \mathrm{f}_{\mathrm{L}}-\mathrm{f}_{2}$. Isolation in the transmission line is assured by the fact that the output IF frequency is different from either of the two input IF's, and microwave isolation at the antenna element is assured by choosing $\mathrm{N}$ so that $N\left(f_{1}-f_{2}\right) \neq f_{1}$ or $f_{2}$. Finally, note that the entire PCC + PRR consists of just five mixers and their assocfated filters and IF limiter amplifiers (not shown), plus one flip-flop $(x$ 1/2). The phase-locked PCC and PRR circuits in Figures 6 and 7 use at least as many mixers and filters as Figure 9, but the latter does not need the phase-locked loop components (phase detector, VCO and loop filter).

\section{Pointing Errors in ARA's}

\section{a) Doppler Errors}

The principle of retrodirectivity requires that the phase delay between two points, $A$ and $B$, be the same for a signal traveling from $A$ to $B$ as $i t$ is from $B$ to $A$. But this symmetry fails when $B$ moves relatively $\div 0 A$; if $\tau_{A B}(t)$ is the phase delay observed at $B$ at time, $t$, of a signal sent from $A$, and $\tau_{B A}(t)$ is the phase delay observed at $A$ at the same time, $t$, of a signal sent from $B$, then $\tau_{A B}(t) \neq \tau_{B A}(t)$, because

$$
\tau_{B A}(t)=\frac{r\left(t-\tau_{B A}(t)\right)}{c},
$$

whereas

$$
\tau_{A B}(t)=\frac{r(t)}{c} \text {. }
$$

If the radial component of the velocity of $B$ relative to $A$ is $v$, then

$$
\tau_{B A}(t)=\frac{\tau_{A B}(t)}{1+\frac{v}{c}} .
$$


To be relativistically precise, we must add that these equations are correct only if the observers at both $A$ and $B$ are at rest with respect to $A$, but since the corrections for moving observers is of order $(v / c)^{2}$, they are negliglble compared to doppler errors which are always of order v/c.

Doppler errors arise in two ways: first, from the radial velocity, $v_{0}$, of the reference element with respect to the pilot source, and second, from the difference, $v_{k}-v_{0}$, between the radial velocities of the $k$ th and reference elements. In Appendix $A$ we have derived an expression for $\delta \phi_{j k}$, the difference in phase between the contributions to the field at the pilot source, as a function $v_{0}, v_{j}$ and $v_{k}$ for $j, k=1, \ldots, n$. Perfect retrodirection is, of course, equivalent to $\delta \phi_{j k}=0$ for all pairs of elements, $j$ and $k$.

Considering first the error due only to the radial motion of the reference element (i.e. setting $v_{j}=v_{k}=v_{0}$ in the expression for $\delta \phi_{j k}$ in Appendix A), we get

$$
\delta \phi_{j k}=\left(\frac{2 \omega v_{o}}{c}\right)\left(\tau_{k}-\tau_{j}\right),
$$

where

$$
\tau_{i}=\left(\frac{1}{c}\right) r_{i}\left(t-\tau_{i}\right), \quad i=j, k,
$$

is the phase delay time for the signal from the $i$-th element arriving at the pilot source $(r=0)$ at time $t$. For a planar array,

$$
\tau_{k}-\tau_{j}=\left(\frac{1}{c}\right) a_{j k} \sin \theta
$$


where $a_{j k}$ is the distance between the $f t h$ and $k t h$ elements, and $\theta$ is the scan angle, 1.e. the angle of incidence of the pllot signal (Figure 10). In Appen$\mathrm{d}$ ix $A$ we show that the transmitted beam points in direction $\theta^{\prime}$ given by

$$
\sin \theta^{\prime}=\left(1+\frac{2 v_{0}}{c}\right) \sin \theta
$$

which gives

$$
\Delta \theta=\theta^{\prime}-\theta=\left(\frac{2 v_{o}}{c}\right) \tan \theta .
$$

Eq. (8) is jusi the squint error, Eq. (4), due to the two way doppler shift,

$$
\Delta \omega=-\left(\frac{2 v_{o}}{c}\right) \omega .
$$

To get some idea of the magnitude of this error, apply (8) to the SPS ARA described in sect. I. Its allowable pointing error is $5.6 \times 10^{-6}$ radians. Therefore, assuming $|\Theta| \leq 45$ degrees (much larger than the scan angles specifled in ARA preliminary designs, (8) gives

$$
\operatorname{Max}\left|\mathrm{v}_{\mathrm{o}}\right|=830 \mathrm{~m} / \mathrm{sec}
$$

The actual radial velocities of geosynchronous satellites are much smaller than this (less than $1.0 \mathrm{~m} / \mathrm{sec}$ in most cases), so we need not worry about "doppler squint" in this rase. Deep space probes, on the other hand, may travel much 
faster than $830 \mathrm{~m} / \mathrm{sec}$ radially, but since they are unlikely to need $1.0 \mathrm{~km}$ diameter ARA's, they can tolerate larger pointing errors.

The expression for the differentia. doppler error is

$$
\Delta \phi_{j k}=\left(\frac{2 \omega}{c}\right)\left(\tau_{k o}\left(v_{0}-v_{k}\right)-\tau_{j o}\left(v_{o}-v_{j}\right)\right)
$$

(the first term of Fq. (A12)), where $\tau_{k o}$ is the transmission line delay between the kth and reference elements. The phase of the kth element's contribution to the field at $r=0$ is

$$
\phi_{k}=\phi_{o}+\delta \phi_{o k}=\phi_{o}+\left(\frac{2 \omega}{c}\right) \tau_{k o}\left(v_{o}-v_{k}\right)
$$

Since it depends on the value of eacin $v_{k}$, rather than just $v_{0}$, the effects of differential doppler are far more various and difficult to calculate than that of simple translational doppler. In the absence of information on the various physIcal properties of the ARA structure and on its attitude control system, we have no way of knowing what steady state or transient motions of the ARA are possible, and therefore, no way of applying (10). The best we can do is to calculate bounds on $\left|v_{o}-v_{k}\right|$ based on a reasonable bound for $r_{k o}$ and an arbitrary $\left|\delta \phi_{\text {ok }}\right|$ bound. A bound for $\tau_{k o}=\ell_{k o} / c_{L}$ is $\tau_{k o} \leq 3 \mathrm{D} / 2 \mathrm{c}$, which results if we assume that $\ell_{\text {ko }} \leq \mathrm{D}$, the dimension of the ARA, and $c_{L} \geq(2 / 3) c$. Let $\delta \phi$ be the allowable phase error. Then from (10),

$$
\left|v_{0}-v_{k}\right| \leq \frac{\lambda c(\delta \phi)}{6 \pi D}
$$

Using the SPS as an example again, $\lambda=0.125 \mathrm{~m}, D=1000 \mathrm{~m}$, and we shall assume $\delta \phi=0.1 \pi \mathrm{rad}$. Then

$$
\left|v_{o}-v_{k}\right| \leq 625 \mathrm{~m} / \mathrm{sec}
$$


is the maximum allowable relative radial velocity of the elements. Such large relative velocities would be most unusual for any spacecraft.

\section{b) Aberration Error}

The transverse component, $v_{T}$, of the ARA relative to the pilot source produces pointing error

$$
\Delta \psi=-\frac{2 v_{T}}{c}
$$

We shal1 call this error "aberration" because it is essentially the same plienomenon as astronomical aberration: the small annual oscillation in the apparent position of stars due to the Earth's orbital motion. Eq. (12) is just twice the astronomical value, as one might expect from the fact that retrodirectivity is a two way light path process, while the light from a star reaches the Earth by a direct, one way, path.

In Figure 11, we assume that the spacecraft carrying the ARA moves with uniform velocity, $v$, with respect to the pilot source, Eq. (12) is obtained by applying the coordinate transformations of special relativity twice: first to obtain the angle of incidence of the pilot signal with respect to the ARA, and second to obtain the angle of incidence of the retrodirected signal with respect to the pilot source. The exact result of the calculation (see Appendix B) includes terms of order $(v / c)^{2}$ and higher, which are dropped to obtain (12).

Aberration error is negligible for geosynchronous satellites. For the $1.0 \mathrm{~km}$ diameter ARA of the SPS, the maximum allowable error, $.56 \times 10^{-5}$ radians, would be exceeded only by

$$
v_{T}=\frac{.56 \times 10^{-5}}{2}\left(3 \times 10^{8}\right)=840 \mathrm{~m} / \mathrm{sec}
$$


whereas $v_{T}<10 \mathrm{~m} / \mathrm{sec}$ for the typical geosynchronous satellite. Even for deep space probes with very large values of $v_{T}$, aberration is not too serious. For example, for missions to the outer planets, $v_{T} \leq 6 \times 10^{4} \mathrm{~m} / \mathrm{sec}$. In order to hold pointing loss to less than $1.0 \mathrm{~dB}$, the pointing error for a uniformly illuminated circular aperture must be less than $\lambda / \pi D$. On this basis, apertures up to

$$
\frac{D}{\lambda} \leq \frac{1}{\pi} \frac{\mathrm{c}}{2 \mathrm{v}}=\frac{3 \times 10^{8}}{(2 \pi) 6 \times 10^{4}} \approx 800 \text { wavelengths }
$$

in diametr: are permissible. The maximum $v_{T}$ for inner planet missions may be somewhat larger than $6 \times 10^{4} \mathrm{~m} / \mathrm{sec}$, but such huge apertures hardly seem necessary for distances less than $2.0 \mathrm{AU}$.

\section{c) Propagation Mediam Effects}

Even if the points $A$ and $B$ are fixed, the required symmetry of phase delay fails if the phase velocity, $v_{A B}$, for propagation from $A$ to $B$, is different from $\mathrm{v}_{B A}$, the $B$ to $A$ phase velocity. This may occur if the phase velocity varies with either time or frequency (since $\mathrm{w}_{\mathrm{AB}} \neq \mathrm{w}_{\mathrm{BA}}$ ). In the case of the SPS, the ionosphere will be the important time varying and dispersive component of the propagation medium. For more distant spacecraft, the solar plasma nady te the dominant medium. The analysis of the effect of the ionosphere on SPS retrodirectivity is greatly complicated by the fact that the ionosphere itself will be significantly affected by energy absorbed from the downlink power beam [11].

\section{d) Effect of Transmission Line Mismatches}

Our previous analysis of central phasing (Subsection IIA) assumed that the phase shift produced by a transmission line of length $\ell$ is simply $-\beta \ell$, for a signal of frequency $\omega=v_{p} \beta$, where the phase velocity, $v_{p}$, is assumed to be independent of $\omega$. But this result does not take into account the effect of 
mismatches at the ends of the line, i.e. at its junctions with the diplexers at either end. When we do so, we find, assuming a wave incident on the left-hand end of the line, that the wave just to the right of the right-hand end is the vector sum of a directly transmitted wave plus the Infinite sum of waves transmitted afte , 2, 3, .. reflections from both mismatches. For a lossless 1ine, the phase of this composite wave is given by

$$
\psi_{a}=-\beta_{a} \rho+\arg \left(T_{1 a} T_{2 a}\right)-\arg \left(1-\Gamma_{1 a} \Gamma_{2 a} \exp \left(-2 j \beta_{a} \ell\right)\right)
$$

at frequency $\omega=v_{p} \beta_{n}$, there $T_{1 a}, \Gamma_{1 a}$ are the transmission and reflection coefficients respectively at the line's left end, and $T_{2 a}, \Gamma_{2 a}$ 'hose at its right end [12], [13]. Obviously, the phase of a leftwards traveling wave just to the left of the left end of the line is also given by (13). Now suppose that the rightgoing wave of fre. ency $\omega_{a}$ is conjugated at the left end of the line and retransmitted down the line to the right at freciency $\omega_{b}$. Then the phase of the conjugated wave just to the right of the right end of the line is

$$
\begin{aligned}
\Delta \phi_{b}(l)= & \psi_{b}-\left(\frac{\omega_{b}}{\omega_{a}}\right) \psi_{a}=\arg \left(T_{1 b} T_{2 b}\right)-\left(\frac{\omega_{b}}{\omega_{a}}\right) \arg \left(T_{1 A} T_{2 a}\right) \\
& -\arg \left[1-\Gamma_{1 b} \Gamma_{2 b} \exp \left(-2 j \beta_{b} \ell\right)\right] \\
& +\left(\frac{\omega_{b}}{\omega_{a}}\right) \arg \left[1-\Gamma_{1 a} \Gamma_{2 a} \exp \left(-2 j \beta_{a} \ell\right)\right] .
\end{aligned}
$$

Because $\mathrm{T}=1+\Gamma$ and $\arg (1)=0$, we find that $\Delta \phi(l)=0$ for $\Gamma_{1 . \mathrm{a}}=\Gamma_{2 \mathrm{a}}=\Gamma_{1 \mathrm{~b}}=$ $\Gamma_{2 b}=0$ (and also for $\omega_{b}=\omega_{a}$, but that impractical case must be excluded). It 
is easy to show (see Appendix c) that, for reasonably sma11 $|\Gamma|$ ( $\Gamma$ denotes any of $\left.\Gamma_{1 a}, \Gamma_{2 a}, \Gamma_{1 b}, \Gamma_{2 b}\right), \Delta \phi_{b}(\ell)$ is bounded by

$$
\left|\Delta \phi_{b}(\ell)\right| \leq\left|\Gamma_{1 b}\right|+\left|\Gamma_{2 b}\right|+\left(\frac{\omega_{b}}{\omega_{a}}\right)\left(\left|\Gamma_{1 a}\right|+\mid \Gamma_{2 a}{ }^{\prime}\right)+\left|\Gamma_{b}\right|^{2}+\left(\frac{\omega_{b}}{\omega_{a}}\right)\left|\Gamma_{a}\right|^{2}
$$

where

$$
\begin{aligned}
& \left|\Gamma_{b}\right|=\operatorname{Max}\left(\left|\Gamma_{1 b}\right|,\left|\Gamma_{2 b}\right|\right), \\
& \left|\Gamma_{a}\right|=\operatorname{Max}\left(\left|\Gamma_{1 a}\right|,\left|\Gamma_{2 a}\right|\right) .
\end{aligned}
$$

Eq. (15) shows that the arg $\left(\mathrm{T}_{1} \mathrm{~T}_{\mathrm{a}}\right)$ terms of (14), which do not depend on $\ell$, are bounded by terms of order $|\Gamma|$, while the last two terms, which do depend on $\ell$, are bounded by $|\Gamma|^{2}$ terms. Thus the variation of the $\Gamma^{\prime}$ 's over the ARA may give rise to phase errors of order $|\Gamma|$, while errors due to different values of $\ell$ for a particular element) are at most of order $|\Gamma|^{2}$. We conclude that the uniformity of the VHF diplexers is more important than the particular values of $|\Gamma|$, assuming, still, that $|\Gamma|$ is reasonably small. Specifically, $|\Gamma| \leq 0.1$ should suffice for uniform diplexers while $|\Gamma| \leq 0.01$ would be required for very nonuniform diplexers. From (14) and (15) we also see that the diplexers should be designed for resistive mismatches to the extent possible, because $\arg \left(\mathrm{T}_{1} \mathrm{~T}_{2}\right)=0$ for real $\Gamma_{1}$ and $\Gamma_{2}$.

\section{e) Multipath Effects}

'lultipath causes pointing errors in much the same way transmission line mis-matches do. The received signal, either pilot or retrodirected, is the vector sum of a direct signal plus one or more signals each of which reach the receiving antenna after one or more reflections from the surroundings. The effect is 
to perturb the phase of the received signal. This, as in the transmission line case, would be harmless if $\omega_{b}=\omega_{a}$, for then reciprocity would assure exactly the same phase perturbation of the retroditected signal received back at the pilot source no matter how complicated the multipath situation. But since $\omega_{b} \neq \omega_{a}$, reciprocity fails and phase errors result. Except in the very simplest cases, multipath effects are too difficult to calculate. Their presence can be inferred, however, by an irregular variation of pointing error with scan angle. We will see such evidence of multipath below in data obtained for a breadboard ARA. Multipath due to scattering from the solar panels may be a significant problem for the SPS ARA.

\section{EXPERIMENTAL RESULTS}

Figure 12 is the block diagram of a two element X-band ARA breadboard which was built and tested by the author at the Jet Propulsiun Laboratory. The purpose of the breadboard is to demonstrate exact phase conjugation and central phasing, both of which, as we saw above, would be necessary features of very large, wtraprecise ARA's.

The PCC in Figure 12 is just that of Figure 6 for $n=4$, so that the frequency translation ratio is $R=2$. Fast MECL flip-flops are used for the $X 1 / 2$ and $\mathrm{X} 1 / 4$ circuits. Since this array has only two elements, both of which must transmit a conjugate signal, one of the elements must serve as reference e] ement both for the other element (the "remote horn" in Figure 12) and for itself ("reference horn"). But, according to (5), the conjugate of the reference fhase is $\phi_{0}^{*}=2\left(2 \phi_{0}-\phi_{0}\right)=2 \phi_{0}$ for $R=2$, so that one needs only a doubler, not a complete PCC, to conjugate $\phi_{0}$. Because of their dissimilarity, the internal phase delays in the doubler and the remote PCC are unequal. A phase shifter at the doubler oucput is used to equalize their overall delays thereby eliminating the pointing 
error which would otherwise exist. No such phase trimming would be required in a practical ARA consisting of many identical PCC'3.

This ARA uses the two pilot tone system described in Figure 8b. The two pilot tones, $\mathrm{f}_{1}$ and $\mathrm{f}_{2}$, are the upper and lower sidebands respectively produced by modulating a carrier at $9675.0 \mathrm{MHz}$ with a phase stable signal at frequency $\mathrm{E}_{\mathrm{R}} / 2$. The two-tone receivers recover the $\mathrm{f}_{1}-\mathrm{f}_{2}=\mathrm{f}_{\mathrm{R}}=49.612005 \mathrm{MHz}$ IF signal uncorrupted by the phase noise of the pilot carrier or the recelver Lo. The conjugated signal at $2 \mathrm{f}_{\mathrm{R}}$ is multiplied up to $\mathrm{X}$-band by a phase-locked $\mathrm{X} 85$ multip1ier whose $\mathrm{f}_{\mathrm{T}}=170 \mathrm{f}_{\mathrm{R}}=8434.04 \mathrm{MHz}$ output is fed to the horn via the $\mathrm{X}$-band diplexer.

The antenna range set-up is shown in Figure 13. The ARA is mounted on the antenna poiftioner at right which rotates only in azimuth. The two ARA elements are identical rectangular horns mounted at the same $2.4 \mathrm{~m}$ height with E planes vertical. The horns look through apertures cut in the large atsorber panel fastened in front of the ARA. The horn separation is $23.2 \lambda_{\mathrm{T}}$, where $\lambda_{\mathrm{T}}=3 \times 10^{10} /$ $8.434 \times 10^{9}=3.55 \mathrm{~cm}$ is the transmitted wavelength. The $E$ and $H$ plane $3 \mathrm{~dB}$ beamwidths of the horns are both about 30 degrees. The rack at left is the pilot source. A test rereiver diplexed to the pilot horn provides the signal for the pattern recorder. The distance between the ARA and the pilot source is about 10m. A considerable amount of absorbing material is required to minimize reflections in this very compact range.

The ARA pattern is shown as the dashed curve in Figure 14; i.e. this curve is the output of the test receiver diplexed to the pilot horn as a function of ARA rotation in azimuth. An interferometer pattern (solid curve), produced by driving both elements from the same source at $\mathrm{f}_{\mathrm{T}}=8434.04 \mathrm{MHz}$, is superimposed on the APA pattern by way of comparison. The actual ARA "pattern," as the term 
is usually used (1.e. the variation of the radiated signal with azimuth angle for a fixed antenna), is sinilar to the interferometer patter 1 . If the ARA were perfectly retrodirective, the lashed curve would be the envelope of the interferometer pattern over the entire scan, but, as we can see, this is true only within about \pm 5 degrees of broadside. The main reason for the increasing departure from perfect retrodirectivity for scan argles greater than 5 degrees is the increase in the multipath contribution to the wignals received by both the ARA horns and the diplexed pilot horn at large scan angles. The effect of multipath is probably aggravated by amplitude to phase conversion in the ARA electronics. This conclusion is qualitatively supported by the fact that retrodirectivity dramatically improved when: 1) the set-up was zeoriented to minimize reflections from nearby buildings, and 2) absorbing paneis and material were placed in front of and on reflecting surfaces as shown in Figure 13.

The central phasing concept is incorporated into the ARA breadboard (Figure 12) by connecting the remote element to its PCC by a coaxial 1ine. The line stretcher included in this coaxial line enables us to make an experimental'check of that concept. The line stretcher is simply a section of coaxial line whose length can be changed mechanically. Hence it is completely bilateral and (at IF) nondispersive. Therefore, apart from the mismatch errors discussed in Subsection IIC(d) above, stretching the line should not affect ARA performance. The results of changing line length are shown in Figure 15. The ARA patterns for the indicated line length changes were recorded in top to bottom time sequence starting with the zero line length change $(\Delta l=0$ in.) pattern as a datum. The near optimum retrodirectivity of the $\Delta \ell=0$ pattern was obtained by trimming the reference channel output phase as explained above. The other two $\Delta l=0$ patterns (between $2 \mathrm{in.}$ and $3 \mathrm{in}$. and between $3 \mathrm{in.}$ and $4 \mathrm{in.)}$ were run as checks on thermal drift. 
We see from Figure 15 that $\Delta l \leq 2$ in. does not appreciably affect the retrodirectivity of the ARA while extending $\Delta l$ to 3 in. almost completely destroys it. The simflarity of the three $\Delta l=0$ patterns shows that thermal drift is not a factor in these measurements. The fact that $\Delta \ell=2 \mathrm{in} .=1.43$ wavelengths at $\mathrm{f}_{\mathrm{T}}=8.434 \mathrm{GHz}$ shows that central phasing is highly effective in splte of the poorly matched rudimentary VHF diplexers used in this breadboard. By means of the expression (derived in Subsection IIC(d) above) for the phase error produced by such mismatches, it car. be shown that these results are consistent with VSWR $\approx 1.5$ for both diplıxers at both frequencies, in rough agreement with the measured VSWR values.

The spikes in the patterns in Figures 14 and 15 look like c: :le slips, probable in auxiliary equipment (signal generator "lock boxes, atc.) rather than in loops in the ARA itself. In any case, they do not affect the validity of the data.

IV. CONCLUSION

Centrally phased ARA's can be used to point the downlink beam of spaceborne antennas which are too large and floppy to be pointed by methods which require structural rigidity (such as mechanical steering or conventional phased array techniques). Antennas for solar power satellites, multiple beam communication satellites, and deep space probes are possible ARA applications. The retrodirectivicy of an ARA is the result of phase conjugation of the pilot signal received by each element of the array. The same principle enables an ARA to simultaneously function as a phased receiving array. Precision pointing as well as inputoutput isolation is provided by exact, frequency translating phase conjugation circuits (PCC's). Pointing errors in ARA's are caused by doppler, aberration, charged particle media, and multipath effects. A two element ARA breadboard, 
using central phasing and exact, frequency translating PCC's, has been built and tested.

Acknowledgments

The author gratefully ackr'wiedges the advice, encouragement and support of Richard Dickinson, Project Manager of the Microwave Power Transmission Project at the Jet Propulsion Laboratory (JPL). A number of the circuit concepts presented here were developed in collaboration with Dr. Robert C. Tausworthe. Thanks are also due Dr. Victo* Galindo-Israel, Dr. Harry Lass, and John W. MacConnell for good advice and criticism, and to Harold W. Mar1in, and Clifford J. Finnie for assisting wilh the erperimental work. All of the above are with JPL. 


\section{REFERENCES}

[1] M.I. Skolnick and D.D. King, "Self-phasing Array Antennas," IEEE Transaction on Antennas and Propagation, vol AP-12, pp. 142, footnote; March, 1964.

[2] B.A. Sichelstiel, W.M. Waters and T.A. Wild, "Self-focusing Array Research Model," IEEE Transactions on Antennas and Propagation, vol. AP-12, pp. 150-154; March, 1964.

[3] C.Y. Pon, "Retrodirective Array Using the Heterodyne Technique," IEEE Transactions on Antennas and Propagation, vol. AP-12, pp. 176-180; March, 1964.

[4] P.E. Glaser, "Power from the Sun: its Future," Science, vol. 162, pp. 857, 861, Nov. 22, 1968.

[5] W.C. Brown, "Satellite Power Stations: a New Source of Energy?" IEEE Spectrum, vol. 10, pp. 38-47; March, 1973.

[6] F.C. Hansen, "Special Issue on Active and Adaptive Antennas," IEEE Iransactions on Antennas and Propagation, vol. AP-12, pp. 140-141; March, 1964.

[7] D.L. Margerum, "Self Phased Arrays," in Microwave Scanning Antennas, Vo1 III, Array Systems. Academic Press, NY, 1966; pp. 347-350.

[8] In [7], pp. 343-344.

[9] In [7], p. 344, Figure 3.

[10] R.C. Tausworthe, Theory and Practical Design of Phase-Locked Receivers, Vo1. I, Technical Report No. 32-819, Jet Propulsion Laboratory, 1966; p. 37.

[11] Microwave Power Transmission Studies, Vo1 III of Space-Based Solar Power Conversion and Delivery Systems Study, ECON, Inc., Princeton, NJ, and Raytheon Co., Wayland, MA; report prepared by Marshall Space Flight Center, NASA. 
[12] R.W. Beatty and T.Y. Otoshi, "Effect of Discontinuities on the Group Delay of Microwave Transmission Line," IEFE Transactions on Microwave Theory and Techniques, Vo1. MTT-23, pp. 919-923, Nov., 1975.

[13] S.P. Cohn and N.P. Weinhouse, "An Autcmatic Microwave Phase Measurement System," Microwave J., vo1. 7, pp. 49-56, Feb, 1965.

[14] W.K. Panofsky and M. Phillips, Classical Electriclty and Magnetism, 2nd Ed., Cambridge, Mass., Addison-Wesley, 1962; pp. 301-303. 
TABLE I. PHASE TABLE FOR FIGURE 9

$$
\Phi_{0}=\left(\omega_{1}-\omega_{2}\right)\left(t-t_{0}\right), \Phi_{1}=\left(\omega_{1}-\omega_{2}\right)\left(t-t_{1}\right)
$$

(1a) $\omega_{1}\left(t-t_{0}\right), \tau_{0}=r_{0} / v$

(1b) $\omega_{2}\left(t-\tau_{0}\right)$

(2) ${ }^{\omega}{ }_{\mathrm{LO}}{ }^{\mathrm{t}+\Phi} \mathrm{LO}$

(3) $\left(\omega_{1}-\omega_{L O}\right) t-\omega_{1} \tau_{0}-\Phi_{L O}$

(4) $\left(\omega_{L O}-\omega_{2}\right) t+\omega_{2} \tau_{0}+\Phi_{L O}$

(5) $3+4=\left(\omega_{1}-\omega_{2}\right)\left(t-\tau_{0}\right)=\Phi_{0}$

(6) $\omega_{1}\left(t-\tau_{1}\right)$

(6b) $\omega_{2}\left(t-\tau_{1}\right)$

(7) $\omega_{L 1} t+\phi_{L 1}$

(8) $\left(\omega_{1}-\omega_{L 1}\right) t-\omega_{1} \tau_{1}-\Phi_{L 1}$

(9) $\left(\omega_{L 1}-\omega_{2}\right) t+\omega_{2}{ }^{\tau} 1+\phi_{L 1}$

(10) $\left(\omega_{1}-\omega_{L 1}\right)\left(t-\tau{ }_{10}\right)-\omega_{1} \tau_{1}-\Phi_{L 1}$

(11) $\left(\omega_{L 1}-\omega_{2}\right)\left(t-\tau_{10}\right)+\omega_{2} \tau_{1}+\phi_{L 1}$

(12) $=(5)=\left(\omega_{L 1}{ }^{-\omega_{2}}\right) t-\left(\omega_{1}-\omega_{2}\right) \tau_{0}+\left(\omega_{1}-\omega_{L 1}\right) \tau_{10}+\omega_{1} \tau_{1}+\Phi_{L 1}$

(13) (5) (11) $=\left(\omega_{1}-\omega_{L 1}\right) t-\left(\omega_{1}-\omega_{2}\right) \tau_{0}+\left(\omega_{L 1}-\omega_{2}\right) \tau_{10}-\omega_{2} \tau_{1}-\Phi_{L 1}$

$14=\left(12+13=\left(\omega_{1}-\omega_{2}\right)\left(t+\tau_{1}+\tau_{10}-2 \tau_{0}\right)\right.$

(15) $\left(\omega_{1}-\omega_{2}\right)\left(t+\tau_{1}-2 \tau_{0}\right)=\phi_{1}^{*}$

(16) $=8+15=\left(2 \omega_{1}-\omega_{2}-\omega_{L 1}\right) t-\omega_{2}{ }^{\tau} 1^{-2\left(\omega_{1}-\omega_{2}\right) \tau_{0}-\Phi_{L 1}}$

(17) $=9+16=2\left(\omega_{1}-\omega_{2}\right)\left(t-\tau_{0}\right)$

$18=(1 / 2)(17)=\left(\omega_{1}-\omega_{2}\right)\left(t-\tau_{0}\right)=\Phi_{0}$ 


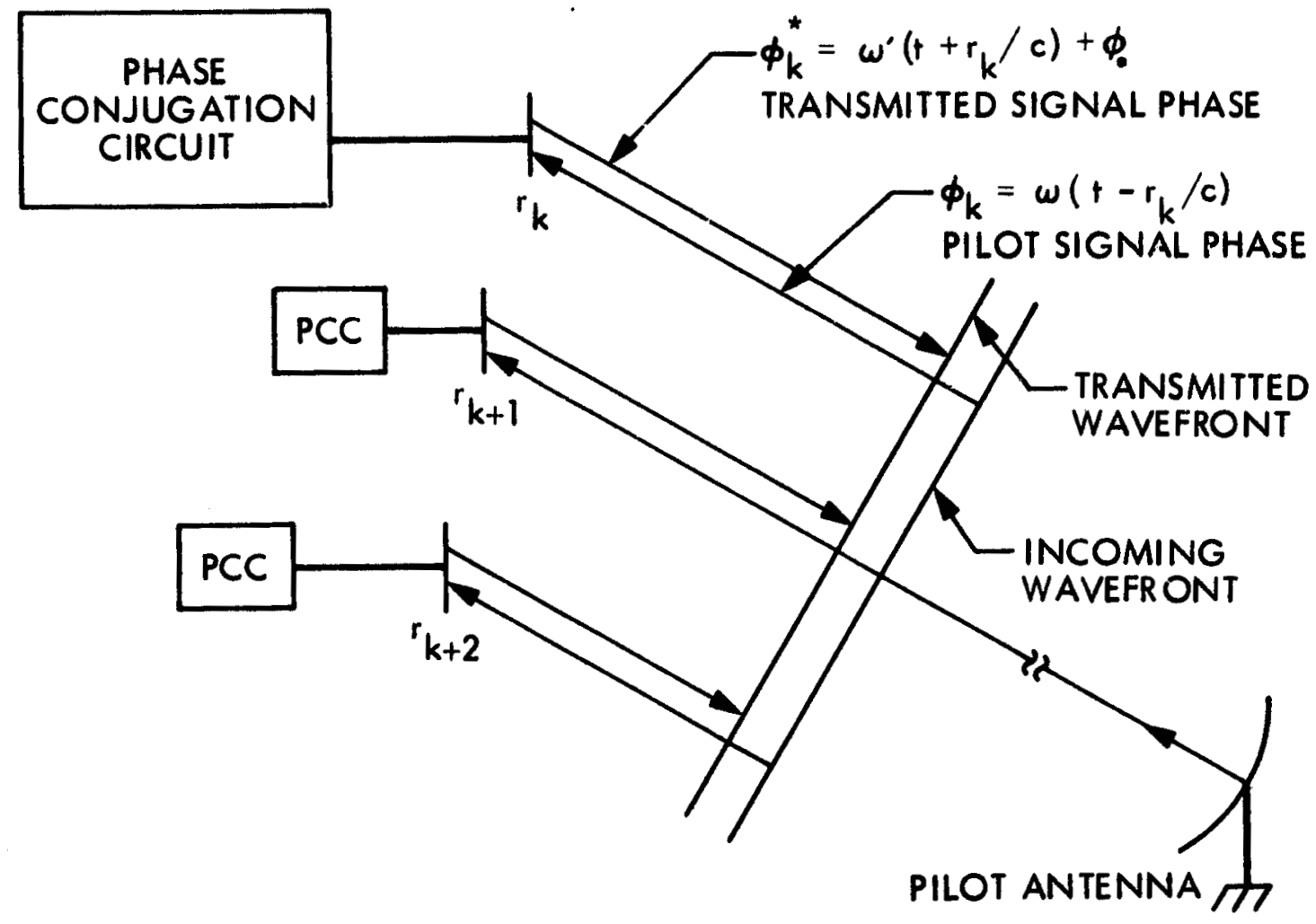

Fig. 1. Active retrodirective array 


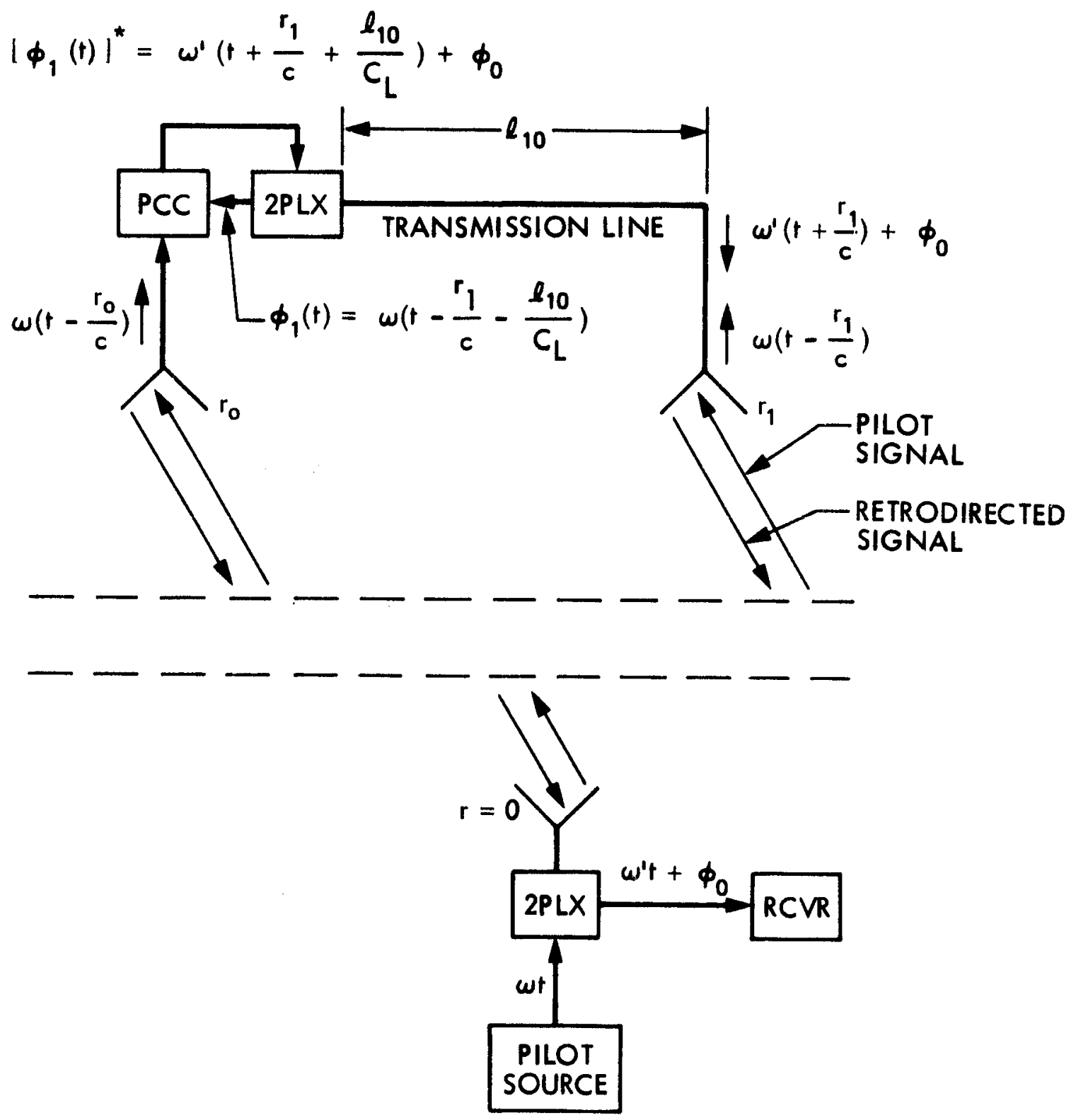

Fig. 2. Central phasing 


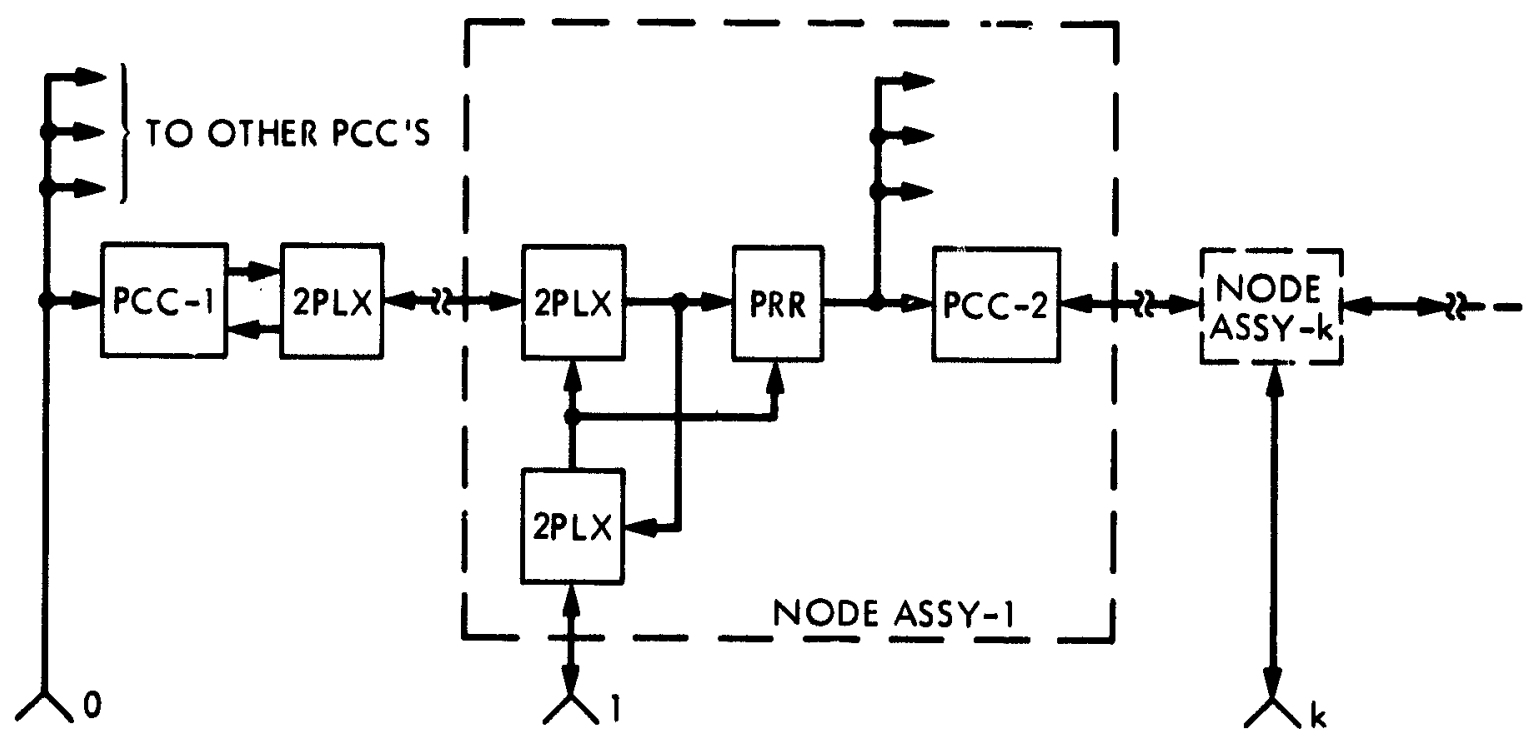

Fig. 3. Tree structure for centrally phased ARA

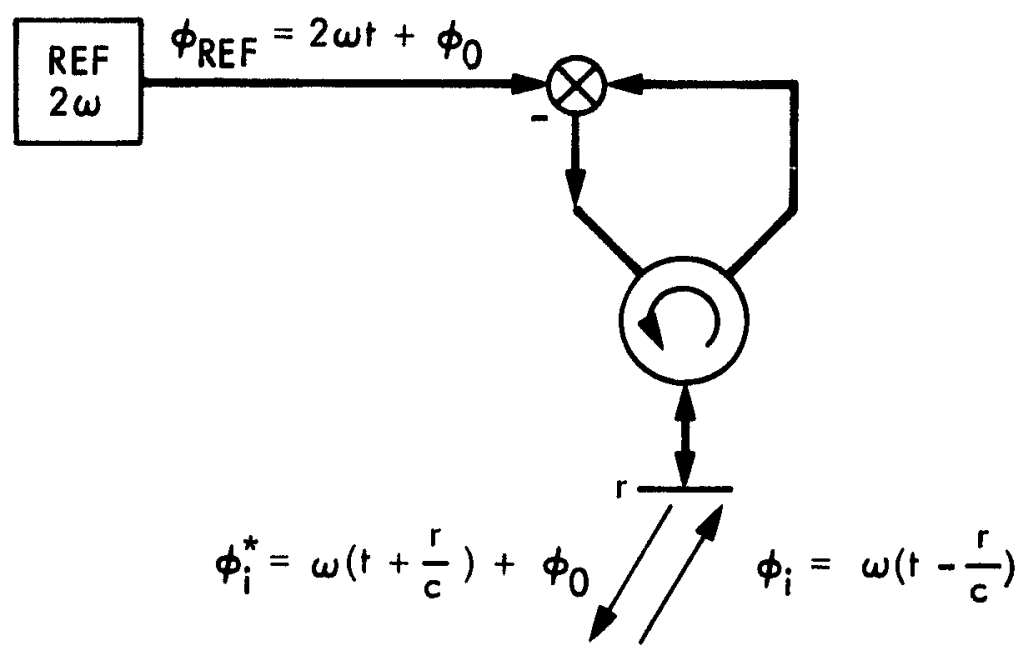

Fig. 4. Simple phase conjugating circuit 


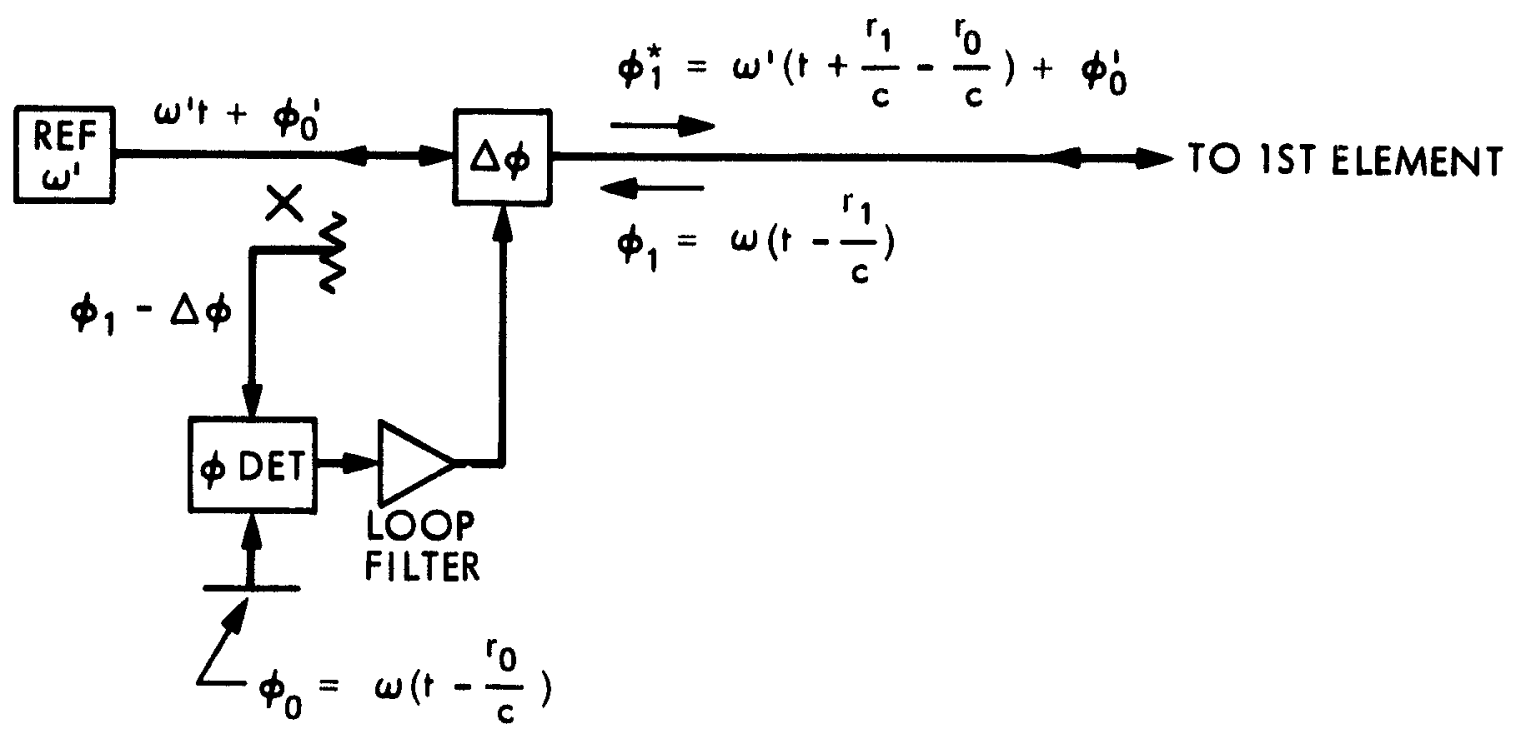

Fig. 5. Exact PCC: servoed $\Delta \phi$ type

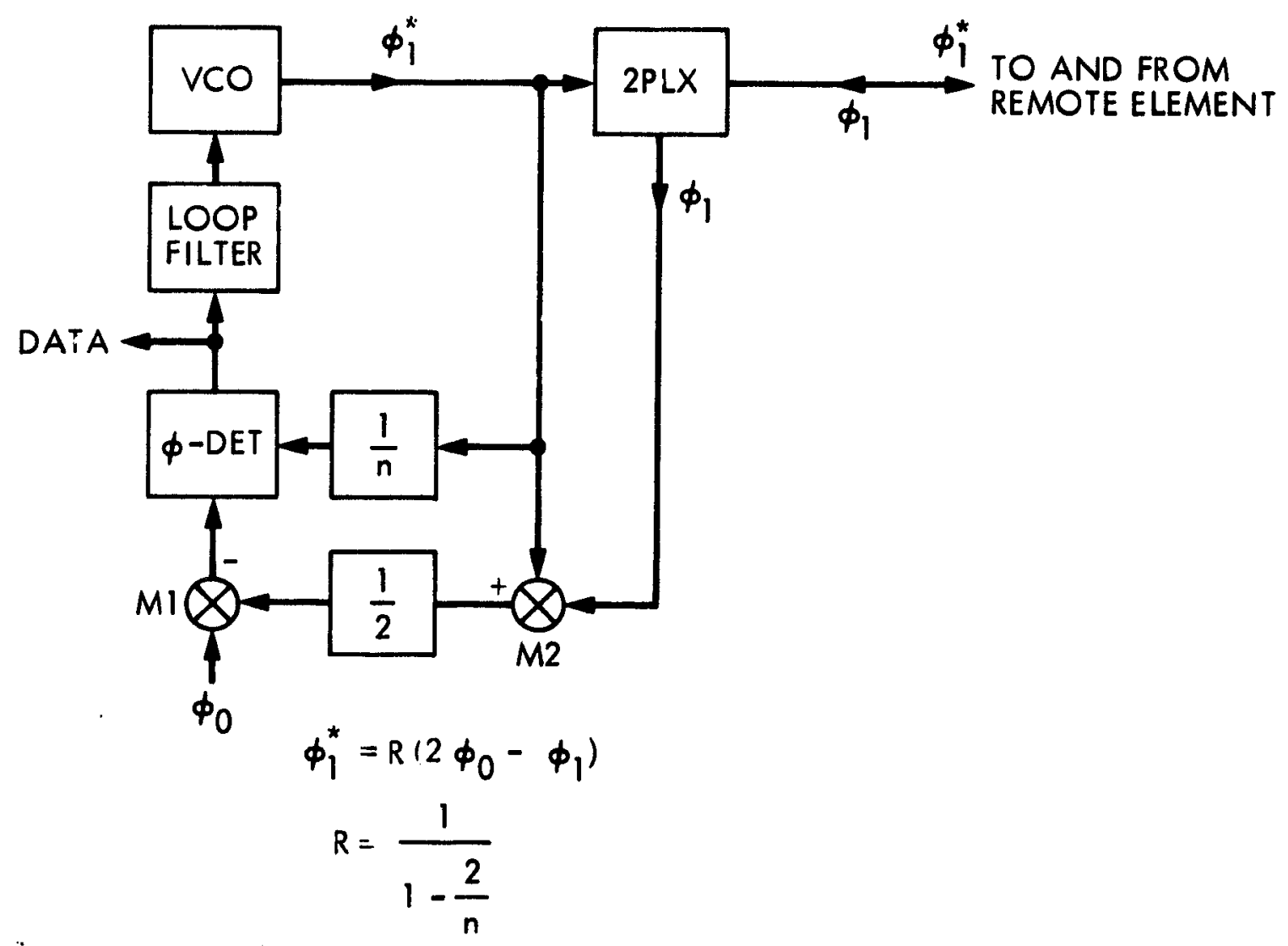

Fig. 6. Exact PCi: Phase locked loop type 


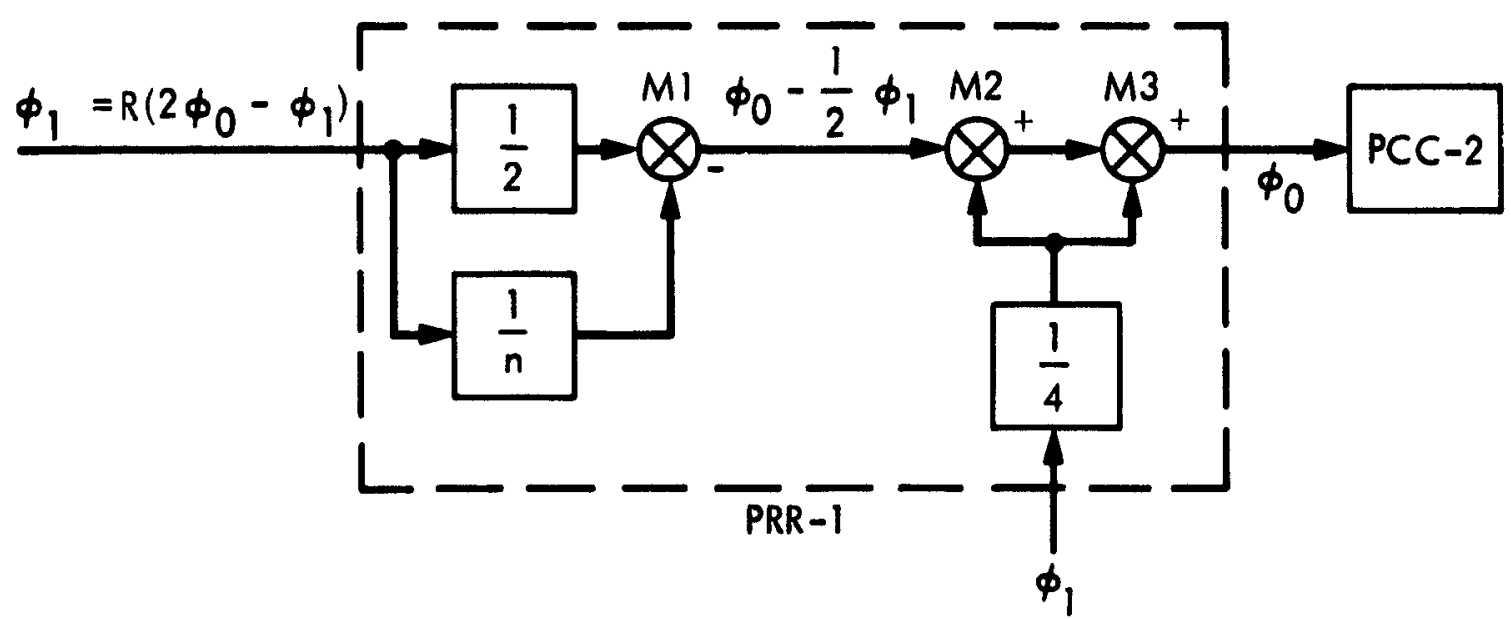

(a)

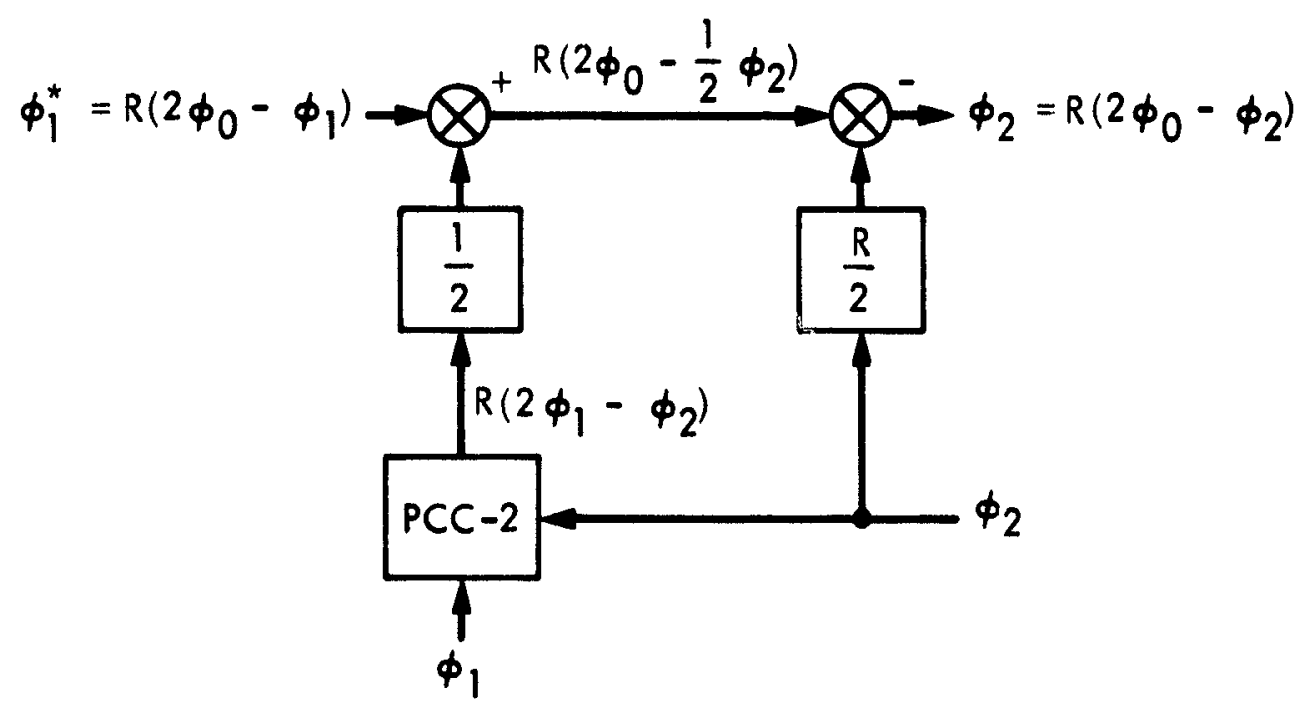

(b)

Fig. 7. Phase reference regenerators (PRRs) for PCC of Figure 6 


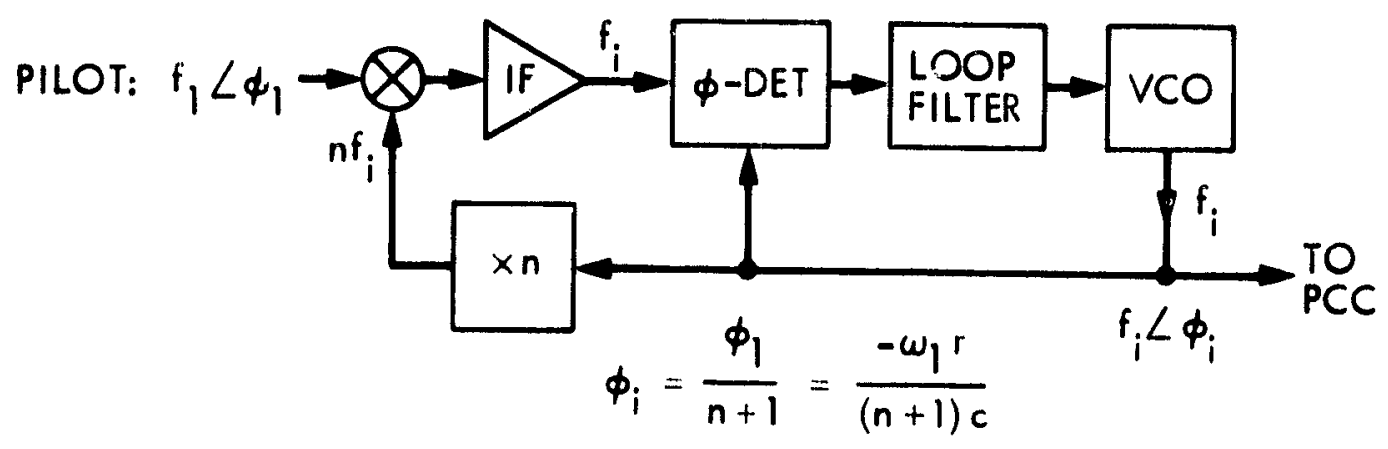

PHASE LOCKED LOOP RECEIVER

(a)
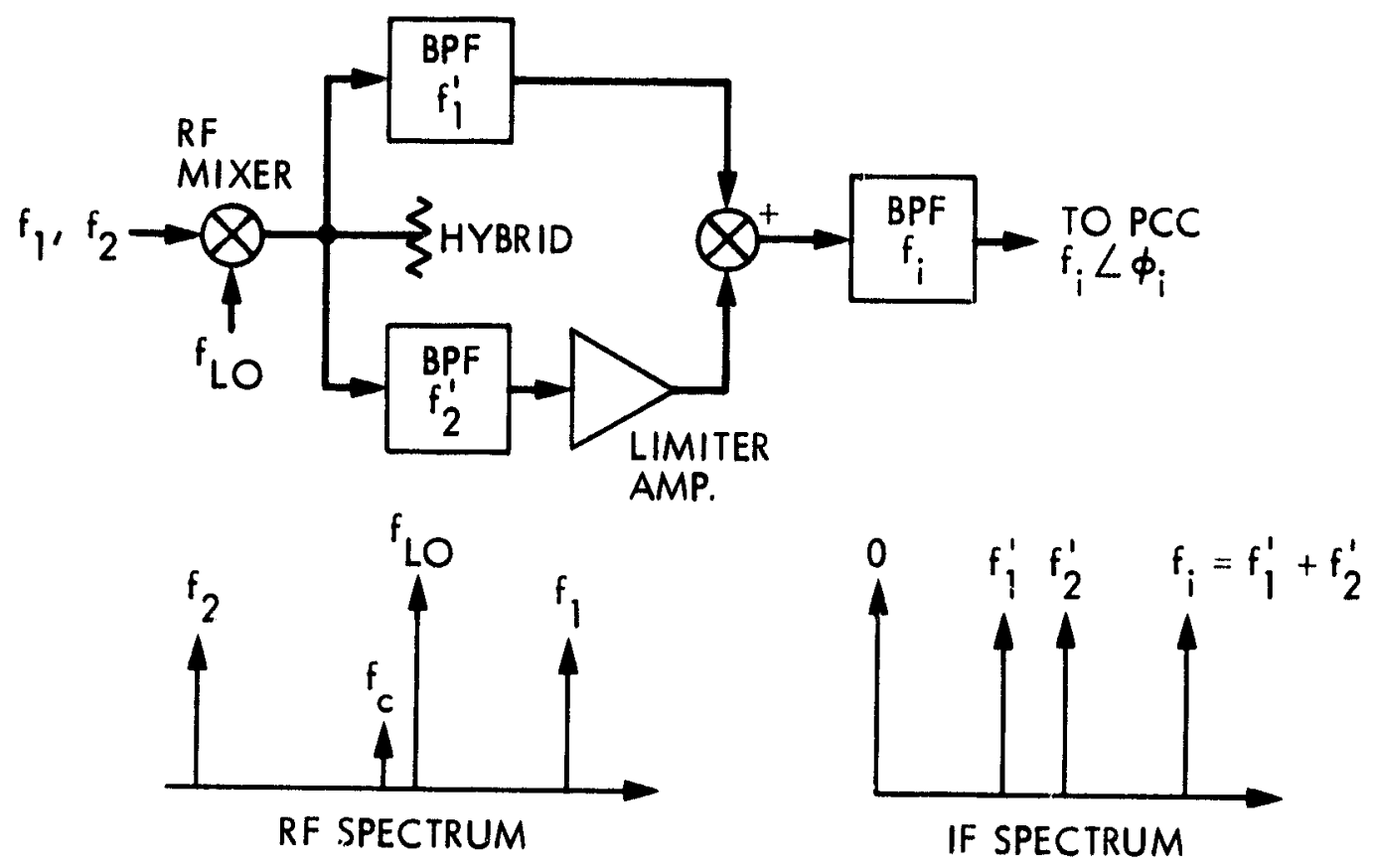

$$
\begin{aligned}
& f_{1}^{\prime}=f_{1}-f_{L O} \\
& f_{2}^{\prime}=f_{L O}-f_{2} \\
& f_{i}=f_{1}^{\prime}+f_{2}^{\prime}=f_{1}-f_{2} \\
& \phi_{i}=\phi_{1}-\phi_{2}=\left(\omega_{2}-\omega_{1}\right) \frac{r}{c}
\end{aligned}
$$

TWO TONE RECEIVER

(b)

FIg. o. ARA receivers 


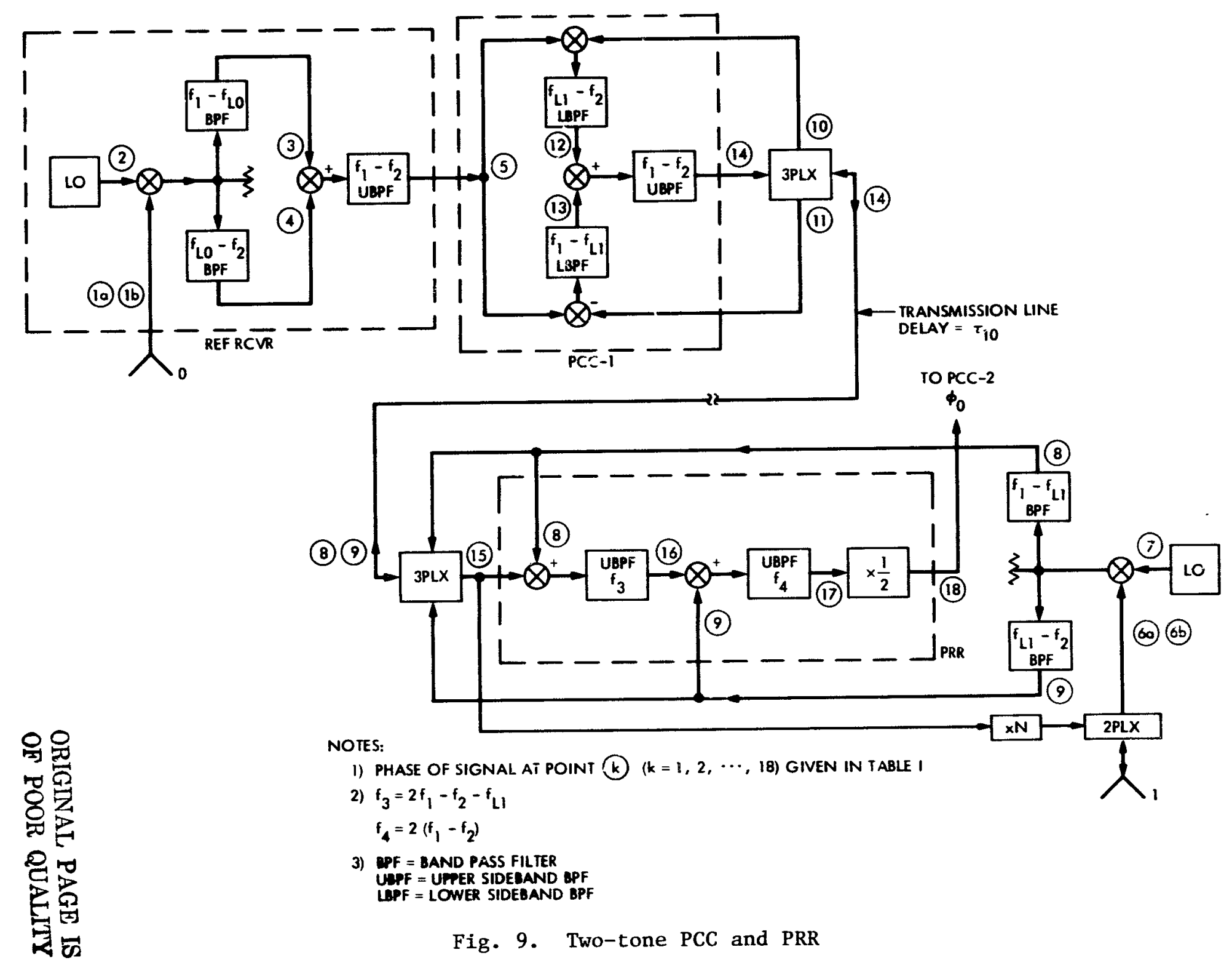




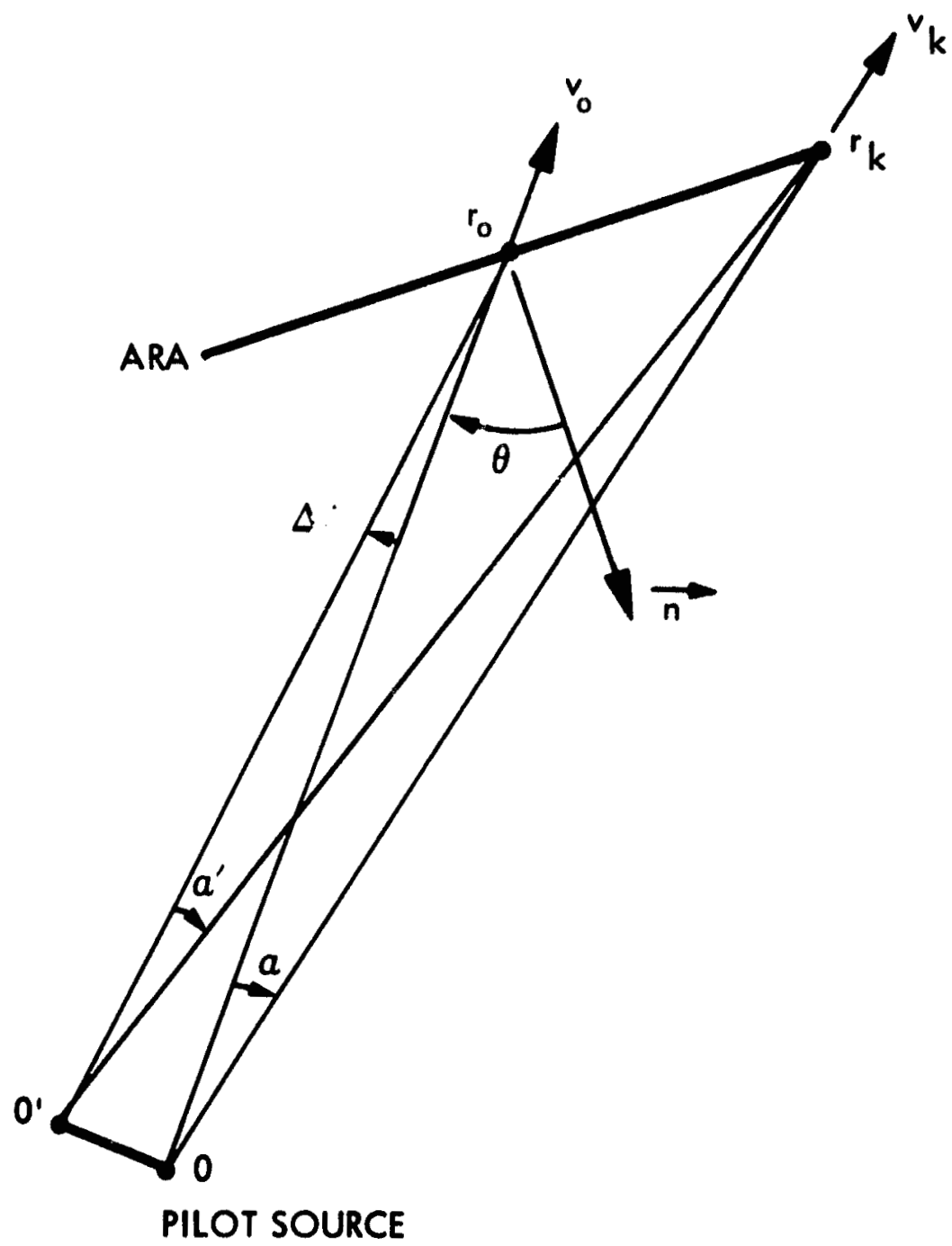

Fig. 10. Doppler pointing error

36 


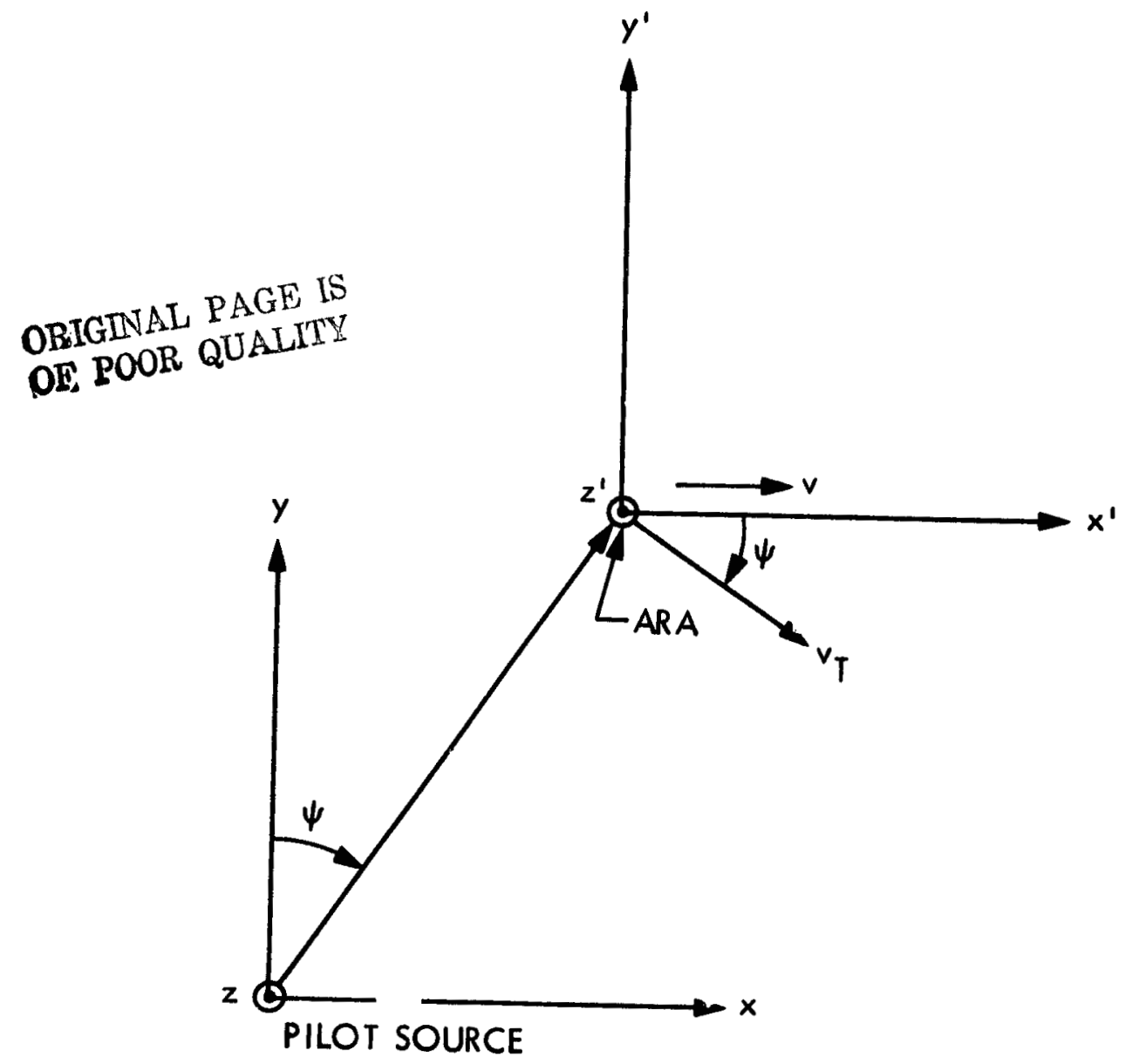

Fig. 11. Aberration pointing error 


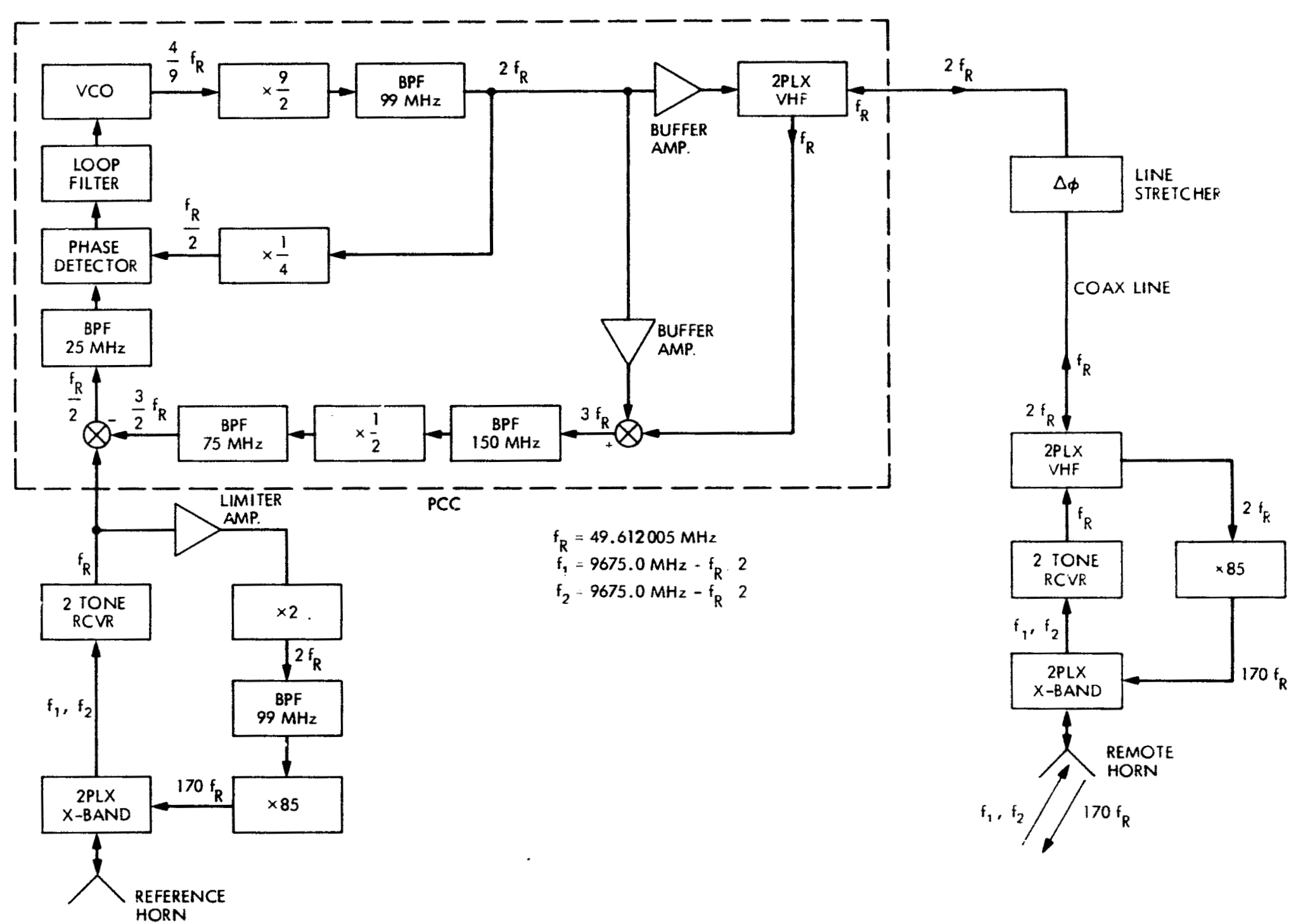

Fig. 12. ARA breadibūard 


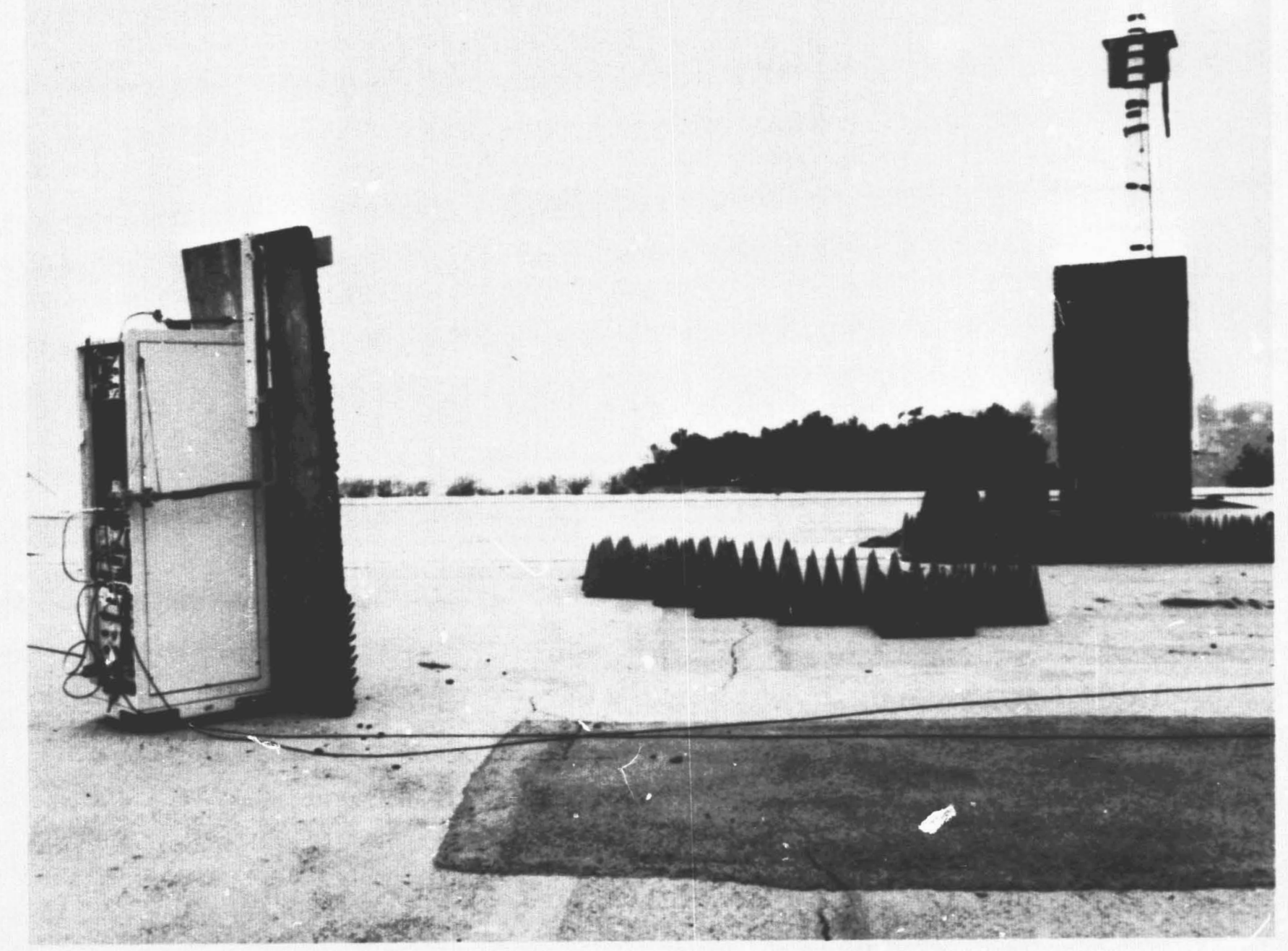

Fig. 13. Antenna range set-up 


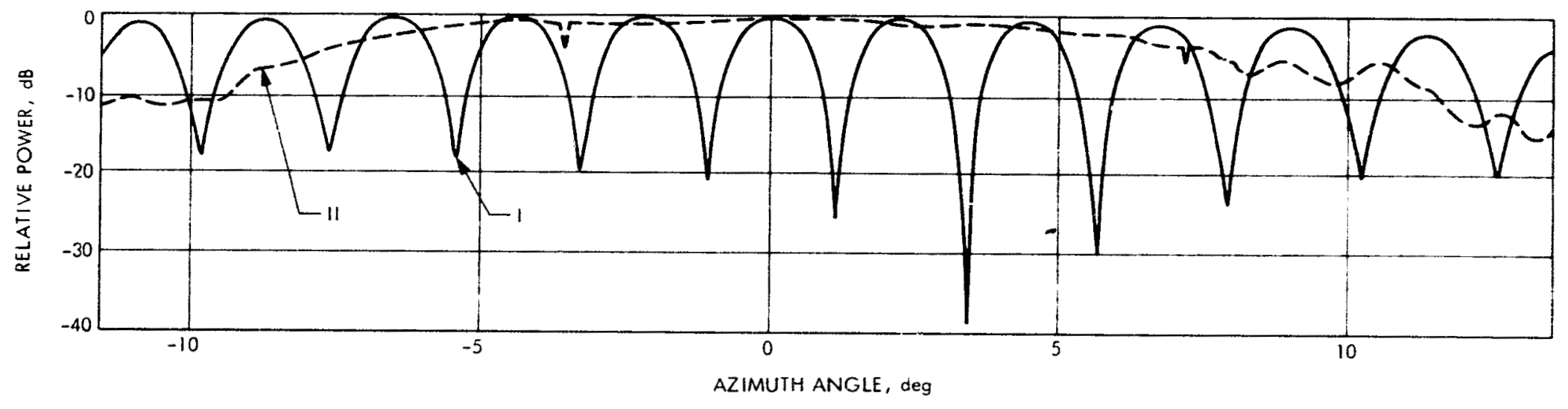

LEGEND:

f
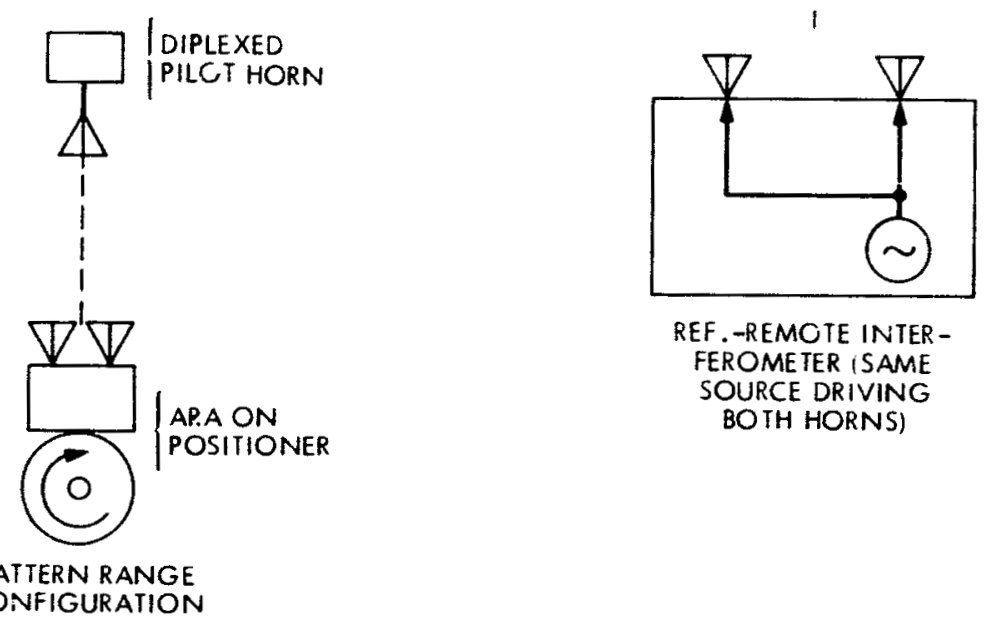

REF. -REMOTE INTER FEROMETER ISAME SOURCE DRIVING BOTH HORNS) CONFIGURATION

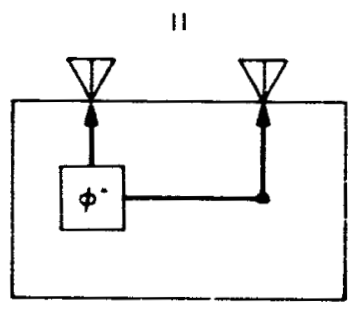

ACTIVE, RETRODIRECTIVE ARRAY ADDED PHASE CONJUGATOR CIRCUITRY

Fig. 14. Retrodirective array pattern 


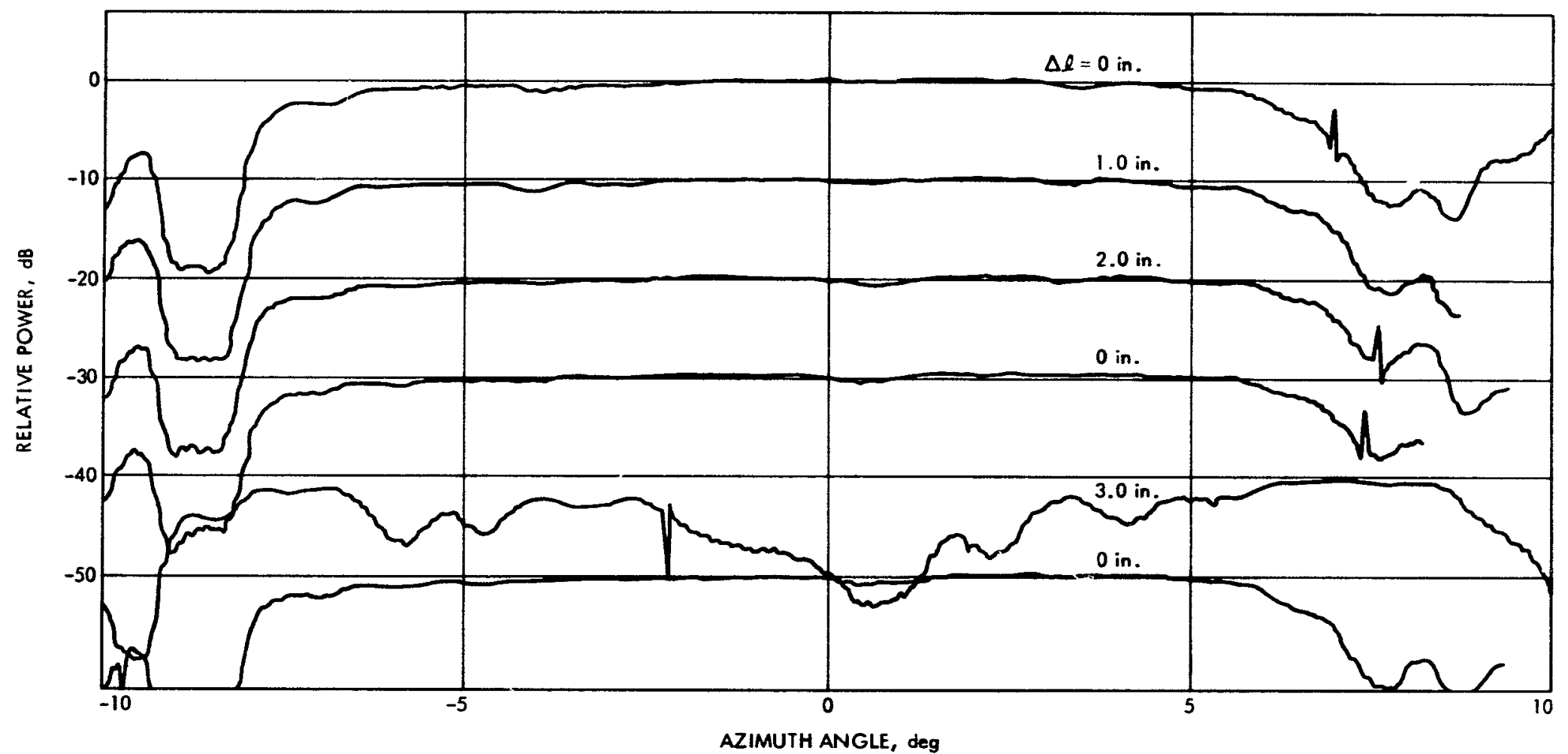

Fig. 15. ARA breadboard: effect on line length changes 


\section{APPENDIX A}

\section{DOPPLER EFFECTS IN ACTIVE RETRODIRECTIVE ARRAYS}

We wish to calculate the phase error in the retrodirected signal produced by the radial component of the motion of the individual elements of the ARA. Accordingly, we denote the distance from the pllot source to the kth element of the ARA by $r_{k}(t)$ where $t$ is time. We assume that the array is centrally phased. Let $k=0$ denote the reference element, and $\rho_{\text {ko }}$ the length of the transmission line between the $k$ th element and its PCC located at the reference element. We also assume, to simplify the algebra of our analysis, that $\rho_{k o}$ s unlike $r_{k}(t)$, is fixed. We show later that this assumption does not greatly affect our result.

The plan of our analysis is to calculate the phase of the conjugated signal. transmitted by the $k t h$ element as seen by a receiver at the pilot source. To do this we calculate the phase of the signal at each stage of its round trip: from the pilot source to the kth element, down the transmission line to the kth PCC, and, after conjugation, back via the same path to the receiver at the pilot source. We then derive an expression for $\delta \phi_{j k}$, the phase difference between the conjugated signals arriving at the pilot source from two different elements, the $j$ th and kth. Finally, we calculate upper bounds for $\left|\delta \phi_{j k}\right|$ as a function of the radial velocities of the elements. Since a perfectly retrodirective array means $\delta \phi_{j k}=0$ for all $j$ and $k,\left|\delta \phi_{j k}\right|$ is a measure of the phase error due to element motion.

The phase of the pilot signal at time $t$ at the pilot source $(r=0)$ is $\omega t$. Therefore, the phase of the pilot signal received by the kth element at time $t$ is

$$
\phi_{k}(t)=\omega\left(t-\left(\frac{1}{c}\right) r_{k}(t)\right)
$$


where $c \approx 3 \times 10^{8} \mathrm{~m} / \mathrm{sec}$. This signal is transmitted to the PCC associated with the kth element via the transmission line of length $\rho_{k 0}$. Let ${ }_{k o}=p_{k o} / C_{L}$ where $C_{L}$ is the phase velocity in the transmission line. The phase, at time $t$ of the input to the kth PCC is obtained from (1) simply by subtracting the phase delay T ko from t throughout $(A-1)$ thus

$$
\phi_{k(1)}(t)=\omega\left(t-\tau_{k o}-\frac{r_{k}\left(t-\tau_{k o}\right)}{c}\right) .
$$

The reference phase at time $t$ is

$$
\phi_{0}(t)=\omega\left(t-\frac{r_{0}(t)}{c}\right) .
$$

Note that the input and reference frequencies, $\dot{\phi}_{k(i)}(t)$ and $\dot{\phi}_{0}(t)$ respectively, are not, in general, the same since we do not assume $\dot{r}_{k}(t)=\dot{r}_{0}(t)$.

We describe the operation of the PCC by the equation

$$
[k(i)]^{*}=R\left[2 \phi_{0}(t)-\phi_{k(i)}(t)\right] \text {, }
$$

where $R>0$ is the transponding ratio. (A4) describes any PCC which uses as its phase reference the pilot signal recelved by one of the elements of the ARA. Substituting (A2) and (A3) into (A4) gives

$$
\begin{gathered}
{\left[\phi_{k(i)}(t)\right]^{*}=\omega^{\prime}\left(t+\tau_{k o}+\frac{r_{k}\left(t-\tau_{k o}\right)-2 r_{0}(t)}{c}\right),} \\
\omega^{\prime}=R \omega .
\end{gathered}
$$


This conjugated signal is now transmitted back to the kth element which in turn retransmits it to the ground. The phase of the conjugated signal at the kth element at time $t$ is obtained from (A5) in exactly the same way we obtained (A2) from (A1), viz. by subtracting $\tau_{\text {ko }}$ from $t$ throughout (A5). Thus

$$
\begin{aligned}
{\left[\phi_{k}(t)\right]^{*} } & =\left[\phi_{k(i)}\left(t-\tau_{k o}\right)\right]^{*} \\
& =\omega^{\prime}\left(\left(t+\tau_{k o}-\tau_{k o}+\left(r_{k}\left(t-2 \tau_{k o}\right)-2 r_{o}\left(t-\tau_{k o}\right)\right) / c\right)\right) \\
& =\omega^{\prime}\left(t+\left(\frac{1}{c}\right)\left(r_{k}\left(t-2 \tau_{k o}\right)-2 r_{o}\left(t-\tau_{k o}\right)\right)\right) .
\end{aligned}
$$

The phase of the conjugated signal arriving back at the pilot source $(r=0)$ at time $t$ is

$$
\begin{aligned}
{\left[\phi_{k}(t, r=0)\right]^{*}=} & {\left[\phi_{k}\left(t-\tau_{k}\right)\right]^{*} } \\
= & \omega^{\prime}\left(t-\tau_{k}+\frac{1}{c}\left(r_{k}\left(t-2 \tau_{k o}-\tau_{k}\right)\right.\right. \\
& \left.\left.-2 r_{o}\left(t-\tau_{k o}-\tau_{k}\right)\right)\right),
\end{aligned}
$$

where

$$
\tau_{k}=\frac{r_{k}\left(t-\tau_{k}\right)}{C},
$$

i.e. $\tau_{k}$ is the "downlink" phase delay of the path between the $k t h$ element and the pilot source at time $t$. Note that it differs from the uplink delay, $r_{k}(t) / c$, in (AI) due to the motion of the $k t h$ element during the interval $\tau_{k}$. 
Let $D=$ the diameter of the ARA. Unless the transmission lines folluw very circuitous routes, $p_{k o} \leq D$. For most transmission 1 ines, $C_{L} \therefore 2 c / 3$. If $D=$ $10^{3} \mathrm{~m}$

$$
\tau_{h o}=\frac{\rho_{k o}}{C_{L}} \leq \frac{10^{3}}{2 \times 10^{8}}=\frac{1}{2} \times 10^{-5} \mathrm{sec} .
$$

This is certainly small enough to justify the approximation

$$
\begin{aligned}
& r_{k}\left(t-\tau_{k}-2 \tau_{k o}\right) \varkappa r_{k}\left(t-\tau_{k}\right)-2 v_{k} \tau_{k o} \\
& =c \tau_{k}-2 v_{k} \tau_{k o}
\end{aligned}
$$

where $v_{k}=\dot{r}_{k}\left(t-\tau_{k}\right)$ is the radial velocity of the kth element at time $t-\tau_{k}$. Similarly,

$$
r_{0}\left(t-\tau_{k}-\tau_{k o}\right)=r_{o}\left(t-\tau_{k}\right)-y_{o} \tau_{k o},
$$

where $v_{0}=\dot{r}_{0}\left(t-\tau_{k}\right)$. Applying (A9) and (A10) to (A7) gives

$$
\begin{aligned}
\phi_{k}(t, r=0) * & =\omega^{\prime}\left(t-\tau_{k}+\left(\frac{1}{c}\right)\left(c \tau_{k}-2 v_{k} \tau_{k o}-2 r_{o}\left(t-\tau_{k}\right)+2 v_{o} \tau_{k o}\right)\right) \\
& =\omega^{\prime}\left(t+\left(\frac{2}{c}\right)\left(\tau_{k o}\left(v_{o}-v_{k}\right)-r_{o}\left(t-\tau_{k}\right)\right)\right) .
\end{aligned}
$$

From (A11) we see that the phase difference, $\delta_{\psi}{ }_{j k}$, between the signals arriving at $r=0$ at time $t$ consists of two terms.

$$
\begin{aligned}
\phi_{k}(t, 0) *-\phi_{j}(t, 0) *=\delta \phi_{j k}= & \left(\frac{2 \omega^{\prime}}{c}\right)\left(\tau_{k 0}\left(v_{0}-v_{k}\right)-\tau_{j o}\left(v_{0}-v_{j}\right)\right) \\
& +\left(\frac{2 \omega^{\prime}}{c}\right)\left(r_{0}\left(t-\tau_{j}\right)-r_{0}\left(t-\tau_{k}\right)\right) .
\end{aligned}
$$


Clearly, $\left|\tau_{k}-\tau_{j}\right| \leq D / c \leq 10^{3} / 3 \times 10^{8}=10^{-5} / 3$ for all $j, k=0,1, \ldots, n$. Therefore, the second term in (A12) can be approximated by

$$
\left(\frac{2 w^{\prime}}{c}\right)\left(r_{0}\left(t-\tau_{j}\right)-r_{0}\left(t-\tau_{k}\right)\right) \stackrel{2}{\imath}\left(\frac{2 \omega^{\prime}}{c}\right) v_{0}\left(\tau_{k}-\tau_{j}\right) .
$$

It suffices to consider on $1 \mathrm{y} \delta \phi_{0 \mathrm{k}}$, the phase error with respect to the reference element's contribution to the signal received at the pilot source. Thus (A12) is simplified to

$$
\begin{aligned}
\delta \phi_{0 \mathrm{k}}= & \left(\frac{2 \omega^{\prime}}{c}\right) \tau_{\mathrm{ko}}\left(\mathrm{v}_{\mathrm{o}}-\mathrm{v}_{\mathrm{k}}\right) \\
& +\left(\frac{2 \omega^{\prime} \mathrm{v}_{\mathrm{o}}}{\mathrm{c}}\right)\left(\tau_{\mathrm{k}}-\tau_{\mathrm{o}}\right) .
\end{aligned}
$$

The first term of (A14) represents the "dIfferential doppler" effect, i.e., the phase error due to the radial component of the motion of the other elements with respect to the reference element. The second term of (A14) is the phase error due to the radial velocity, $v_{0}$, of the reference element with respect to the pilot source. Thus the second term represents only translatory motion while the first represents the rotation, nutation, vibration, etc. of the ARA.

If the ARA is planar, the second term causes a pointing error. To see this, consider Figure 10. Let the distance between the $k$ th and reference elements be $\mathrm{a}_{\mathrm{Ok}}$. Then

$$
\delta \phi_{o k}(\text { translation })=\frac{2 \omega^{\prime} v_{o} a_{0 k} \sin \theta}{c^{2}}
$$


We assume that the ARA is so far from the pllot source that angles $\alpha$ and $x^{\prime}$ are infinitesimal. That being so, we may write

$$
r_{k}=r_{0}+a_{0 k} \sin \theta \text {. }
$$

We assume $\theta \neq 0$, hence $\delta \phi_{0 \mathrm{k}} \neq 0$. Therefore, the peak of the dowlink beam, $1 . e .$, the direction, if 1 t exists, for which $\delta \phi_{0 j}=0$ for all $\mathrm{j}$, does not point towards 0 , the pilot source. Let's assume that $\delta \phi_{0 k}=0$ at some other point, 0 ', which is also at distance $r_{0}$ from the reference element but displaced from 0 by the small angle $\Delta \theta$. The distance from the kth element to $0^{\prime}$ is

$$
r_{k}^{\prime}=r_{o}+a_{0 k} \sin \left(\theta+\Delta \theta_{k}\right)
$$

Let $\phi_{k}\left(t, O^{\prime}\right) *$ be the phase of the retrodirected signal from the $k$ th element as observed at $0^{\prime}$. By our assumption,

$$
\phi_{k}(t, 0) *-\phi_{0}\left(t, 0^{\prime}\right) *=0
$$

Since 0 and $0^{\prime}$ are equidistant from the reference element, $\phi_{0}\left(t, 0^{\prime}\right) *=\phi_{0}(t, 0) *$. Therefore,

$$
\begin{aligned}
\delta \phi_{\mathrm{ok}} & =\phi_{k}(t, 0) *-\phi_{0}(t, 0) *-\left(\phi_{k}\left(t, 0^{\prime}\right) *-\phi_{0}\left(t, 0^{\prime}\right) *\right) \\
& =-\frac{\omega^{\prime} r_{k}}{c}+\frac{\omega^{\prime} r_{k}^{\prime}}{c}=\frac{\omega^{\prime} a_{o k}}{c}\left(\sin \left(\theta+\Delta \theta_{k}\right)-\sin \theta\right) .
\end{aligned}
$$

So, by (A15),

$$
\frac{\sin \left(\theta+\Delta \theta_{k}\right)}{\sin \theta}=\left(1+\frac{2 v_{o}}{c}\right) .
$$


The fact that the index $k$ doesn't appear on the rlght side of 20 shows that $\Delta \theta_{k}=\Delta \theta$ is independent of $k$, 1.e., the seam does have a peak and it points towards $0^{\prime}$. Dropping the subscript, and expanding sin $(\theta+\Delta \theta)$ gfves

$$
\Delta \theta=\frac{2 v_{o}}{c} \tan \theta .
$$

We assumed in the foregoing that $r_{k o}$ is fixed, but the case of time varying fko $(t)$ can be handled by a straightforward extension of that analysis. If we do this, (A14) becomes

$$
\begin{aligned}
\delta_{\text {ok }}= & \left(\frac{2 \omega^{\prime}{ }^{\tau}{ }_{k o}}{c}\right)\left(v_{o}-v_{k}+\left(\frac{1}{2}\right) v_{k o}\right) \\
& +\left(\frac{2 \omega^{\prime} v_{0}}{c}\right)\left(\tau_{k}-\tau_{o}\right),
\end{aligned}
$$

where $v_{k o}=\dot{p}_{k o}(t)$. As one would expect, only the differential phase error is affected. 
APPENDIX B

\section{ABERRATION ERROR}

The formula,

$$
\Delta \psi=\frac{2 \mathrm{v}_{\mathrm{T}}}{\mathrm{c}}
$$

for the pointing error of an ARA due to the transverse component, $v_{T}$, of its velocity relative to the pilot source is obtained from the velocity addition formulas of special relativity as follows. Let the pilot source be at rest a'. the origin of the $X, Y, Z$ coordinate system (CS) as shown In Figure 11, and let the spacecraft carrydng the ARA be at rest at the origin of the $X^{\prime}, Y^{\prime}, Z^{\prime} \operatorname{CS}$. We assume that the spacecraft, along with 1 ts CS, moves at constant velocity $v$ with respect to the pilot source and its $C S$, and we orient the spacecraft $C S$ so that the $X^{\prime}$ axis coincides with the velocity vector $\bar{v}(v=|\bar{v}|)$. He also orient the $X, Y, Z$ axes of the pilot source CS parallel to the $X^{\prime}, Y^{\prime}, Z^{\prime}$ axes, respectively, of the spacecraft CS. Finally, for the sake of brevity, let $z=z^{\prime}$ (ihe derivation for the general case, $Z \neq Z^{\prime}$, is a straightforward but repetitious extension of the less general one that follows). The angle between the pilot source to spacecraft vector $\left(0 \rightarrow 0^{\prime}\right)$ and the $Y$ axis is denoted by $\psi$.

The velocity addition equations relating the velocity, $\vec{u}=\left(u_{x}, u_{y}, u_{z}\right)$, of a point in the $X, Y, Z$ (pilot source) $\operatorname{CS}$ to its velocity, $\bar{u}^{\prime}=\left(u_{x}^{\prime}, u_{y}^{\prime}, u_{z}^{\prime}\right)$ in the $X^{\prime}, Y^{\prime}, Z^{\prime}$ (spacecraft) CS are [14]:

$$
\begin{aligned}
& u_{x}^{\prime}=\left(u_{x}-v\right) f\left(u_{x}\right) \\
& u_{y}^{\prime}=u_{y} \frac{f\left(u_{x}\right)}{\gamma}
\end{aligned}
$$




$$
u_{z}^{\prime}=u_{z} \frac{f\left(u_{x}\right)}{\gamma},
$$

where

$$
f\left(u_{x}\right)=\frac{1}{1-\frac{v u_{x}}{c^{2}}} \text {, }
$$

and

$$
\gamma=\left(1-\left(\frac{v}{c}\right)^{2}\right)^{-1 / 2}
$$

The "point" in our case is the pilot signal traveling from 0 to $0^{\prime}$. In order to be able to speak of the velocity of this signal, we must assume that it is a wave train of finite length, but since the length is arbitrary, the result holds for a CW signal. The velocity of the wave train is $c$, the group (and phase) velocity of plane EM waves in empty space (atmospheric and plasma effects are neglected). Therefore, the velocity components in the $X, Y, Z$ CS are:

$$
\begin{aligned}
& u_{x}=c \sin \psi \\
& u_{y}=c \cos \psi \\
& u_{z}=0
\end{aligned}
$$

Applying these to $(B I a$ ) and ( $B 1 b)$ (we drop ( $B I C$ ) since $u_{z}=0$ ), we get

$$
u_{x}^{\prime}=\frac{c \sin \psi-v}{1-\left(\frac{v}{c}\right) \sin \psi}
$$


and

$$
u_{y}^{\prime}=\frac{c}{r} \frac{\cos \psi}{1-\frac{v}{c} \sin \psi} .
$$

These are the velocity coluponents of the pilot signal incident on the ARA as seen from the ARA. The components of the retrodirected signal are, therefore,

$$
\begin{aligned}
& u_{x}^{*}=-u_{x}^{\prime} \\
& u_{y}^{*}=-u_{y}^{\prime}
\end{aligned}
$$

We may now apply the (B1) transformation again to find $u_{x}^{\prime \prime}$ and $u^{\prime \prime}$, , the components of the signal received back at the pilot source from the ARA, but in doing so, note that the velocity of the pilot source relative to the spacecraft CS is $-v$. Therefore,

$$
\begin{aligned}
& u_{x}^{\prime \prime}=\left(u_{x}^{*}+v\right) f\left(u_{x}^{*}\right) \\
& u_{y}^{\prime \prime}=\frac{u_{y}^{*}}{\gamma} f\left(u_{x}^{*}\right)
\end{aligned}
$$

Substituting now from (B2) and (B3) we get

$$
\begin{aligned}
& u_{x}^{\prime \prime}=\left[2 v-\left(1+\left(\frac{v}{c}\right)^{2}\right) c \sin \psi\right] f\left(u_{x}^{*}\right) /\left(1-\frac{v}{c} \sin \psi\right) \\
& u_{y}^{\prime \prime}=-\frac{(c \cos \psi)}{\gamma^{2}} f\left(u_{x}^{*}\right) /\left(1-\frac{v}{c} \sin \psi\right) .
\end{aligned}
$$


Letting $\psi^{\prime \prime}$ be the angle between the $\mathrm{Y}$ axis and the retrodirected ray incident on the pilot source, we have from (B5)

$$
\begin{aligned}
\tan \psi^{\prime \prime} & =\frac{u_{x}^{\prime \prime}}{u_{y}^{\prime \prime}} \\
& =\frac{\left[2 v-\left(1+\left(\frac{v}{c}\right)^{2}\right) c \sin \psi\right] \gamma^{2}}{-c \cos \psi} \\
& =\tan \psi-\frac{2 v}{c} \sec \psi+0\left(\left(\frac{v}{c}\right)^{2}\right) .
\end{aligned}
$$

Assuming that $\Delta \Psi$ is small,

$$
\tan \psi^{\prime \prime}=\tan \psi+(\Delta \psi) \frac{\mathrm{d}(\tan \psi)}{\mathrm{d} \psi}=\tan \psi+(\Delta \psi)(\sec \psi)^{2},
$$

where $\Delta \psi=\psi^{\prime \prime}-\psi$. Solving for the pointing error, $\Delta \psi$, from (B6) and (B7) and dropping $O\left((v / c)^{2}\right)$ terms, we get

$$
\Delta \psi=-\frac{2 \mathrm{v}}{\mathrm{c}} \cos \psi=-\frac{2 \mathrm{v}_{\mathrm{T}}}{\mathrm{c}} .
$$

(B6) and (B7) break down for $\psi$ in the neighborhood of $+\pi / 2$ but we can still get (B8) for this case by writing (B6) and (B7) in terms of $\theta=(\pi / 2)-\psi$ for $\psi$ near $\pi / 2$, or $\theta=-(\pi / 2)-\psi$ for $\psi$ near $-\pi / 2$. Dropping $\left.0(\mathrm{v} / \mathrm{c})^{2}\right)$ terms is justified because $v / c<3 \times 10^{-4}$ for all existing or foreseeable spacecraft. 
APPENDIX C

TRANSMISSION LINL MISAATCH ERRORS: UPPER BOUND FOR $\left|A_{b}(x)\right|$

By $T=1+\mathrm{I}$, we have

$$
\begin{aligned}
\arg \left(\mathrm{T}_{1} \mathrm{~T}_{2}\right) & =\arg \left(\mathrm{T}_{1}\right)+\arg \left(\mathrm{T}_{2}\right)=\arg \left(1+\mathrm{r}_{1}\right)+\arg \left(1+\Gamma_{2}\right) \\
& =\arctan \left(\frac{\left|\Gamma_{1}\right| \sin \theta_{1}}{1+\left|\Gamma_{1}\right| \cos \theta_{1}}\right)+\arctan \left(\frac{\left|\Gamma_{2}\right| \sin H_{2}}{1+\Gamma_{2} \mid \cos \theta_{2}}\right),
\end{aligned}
$$

where $\theta=\arg (\Gamma)$. Maximizing each term with respect to 0 gives

$$
\begin{aligned}
& \arg \left(\mathrm{T}_{1} \mathrm{~T}_{2}\right) \mid \leq \arctan \left[\frac{\left|\Gamma_{1}\right|}{\left(1-\left|\Gamma_{1}\right|^{2}\right)^{1 / 2}}\right]+\arctan \left[\frac{\left|\Gamma_{2}\right|}{\left(1-\left|\Gamma_{2}\right|^{2}\right)^{1 / 2}}\right] \\
& \leq \frac{\left|\Gamma_{1}\right|}{\left(1-\left|\Gamma_{1}\right|^{2}\right)^{1 / 2}}+\frac{\left|\Gamma_{2}\right|}{\left(1-\left|\Gamma_{2}\right|^{2}\right)^{1 / 2}} \\
& =\left|\Gamma_{1}\right|+0\left(\left|\Gamma_{1}\right|^{3}\right)+\left|\Gamma_{2}\right|+0\left(\left|\Gamma_{2}\right|^{3}\right)
\end{aligned}
$$

where $O\left(|\Gamma|^{3}\right)$ denotes a remainder of order $|r|^{3}$.

Similarly,

$$
\mid \arg \left[1-r_{1} r_{2} \exp \left(-2 j(R \ell)|| \leq\left|r_{1} \Gamma_{2}\right|+0\left(\left|r_{1} r_{2}\right|^{3}\right)\right.\right.
$$

Neglecting all terms of order 3 and higher, (15) follows immediately. 